\title{
Sudden Gains in Cognitive-Behavioral Therapy for Eating Disorders
}

Adriane Ito de Queiroz Cavallini

Brigham Young University - Provo

Follow this and additional works at: https://scholarsarchive.byu.edu/etd

Part of the Psychology Commons

\section{BYU ScholarsArchive Citation}

Cavallini, Adriane Ito de Queiroz, "Sudden Gains in Cognitive-Behavioral Therapy for Eating Disorders" (2010). Theses and Dissertations. 2466.

https://scholarsarchive.byu.edu/etd/2466

This Dissertation is brought to you for free and open access by BYU ScholarsArchive. It has been accepted for inclusion in Theses and Dissertations by an authorized administrator of BYU ScholarsArchive. For more information, please contact scholarsarchive@byu.edu, ellen_amatangelo@byu.edu. 
Sudden Gains in Cognitive-Behavioral Therapy for Eating Disorders

Adriane Ito de Queiroz Cavallini

A dissertation submitted to the faculty of

Brigham Young University

in partial fulfillment of the requirements for the degree of

Doctor of Philosophy

Diane L. Spangler, Chair

Scott Baldwin

Patrick Steffen

Michael Larson

Niwako Yamawaki

\author{
Department of Psychology \\ Brigham Young University
}

August 2011

Copyright $₫ 2010$ Adriane Ito de Queiroz Cavallini

All Rights Reserved 


\author{
ABSTRACT \\ Sudden Gains in Cognitive-Behavioral Therapy for Eating Disorders \\ Adriane Ito de Queiroz Cavallini \\ Department of Psychology \\ Doctor of Philosophy
}

The present study examined whether or not the temporal pattern of symptom change defined as sudden gains is applicable to and has significant ramifications for understanding recovery from eating disorders. Sudden gains were defined as stable and clinically significant changes that take place between two sessions of treatment. Data for the current study were drawn from an efficacy study of CBT for eating disorders which included session-by-session measures of eating disorder symptomatology. Predictors of sudden gains were measured by an observer coded scale that included ratings of therapist use interventions, client change in behaviors and beliefs, client engagement, and homework completion.

Three research questions were addressed: First, is the phenomenon of sudden gains present in CBT for eating disorders? Second, do sudden gains in CBT for eating disorders follow the three-stage model proposed for sudden gain recovery in other disorders (i.e., cognitive changes during critical sessions $=>$ sudden gains $=>$ upward spiral that includes further cognitive changes and greater long-term symptom improvement (Tang \& DeRubeis, 1999b)? Third, what are the predictors of sudden gains in CBT for eating disorders that distinguish the critical session that takes place right before the sudden gain?

Findings suggest that many eating disordered clients $(62 \%)$ experienced at least one sudden gain during the course of CBT treatment. Three distinct types of sudden gains were identified: total symptom sudden gains, eating-related sudden gains, and body-related sudden gains. The average magnitude of these sudden gains was large representing on average $35 \%$ of total symptom improvement. Clients who experienced total symptom and body-related sudden gains demonstrated fewer eating disordered symptoms than the other clients at posttreatment. During the session preceding the sudden gain, therapists had increased levels of cognitive interventions and empathy, and clients experienced more cognitive changes and increased motivation.

Keywords: sudden gains, eating disorders, cognitive-behavioral therapy 


\section{TABLE OF CONTENTS}

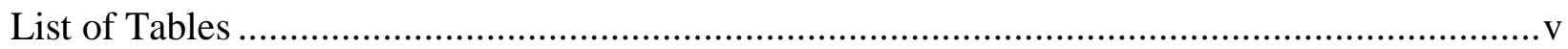

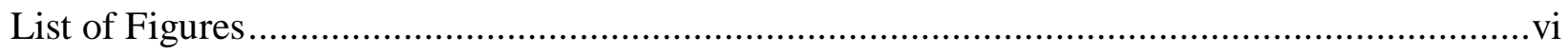

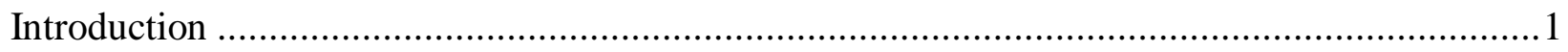

Sudden Gains in CBT for Depression .............................................................. 1

Sudden Gains in Other Therapies for Depression ............................................

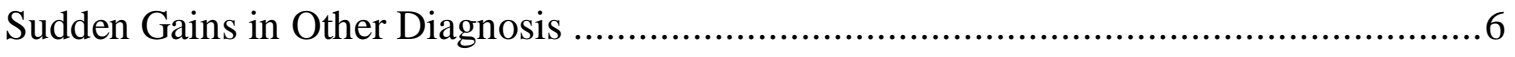

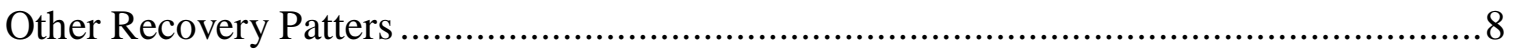

Cognitive-Behavioral Therapy for Eating Disorders......................................... 10

Predictors of Sudden Gains in CBT for Eating Disorders .................................... 13

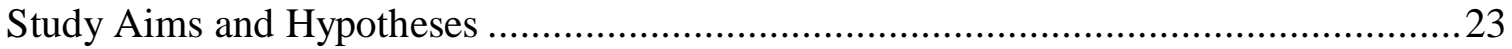

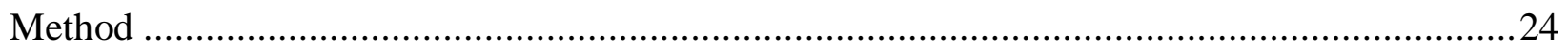

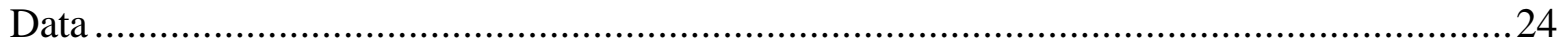

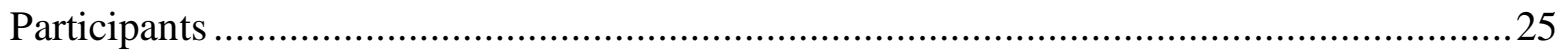

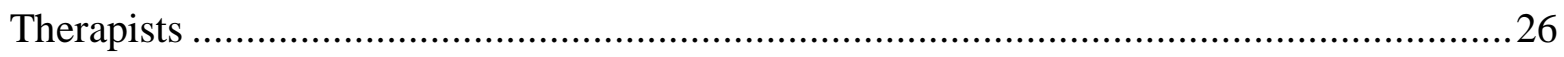

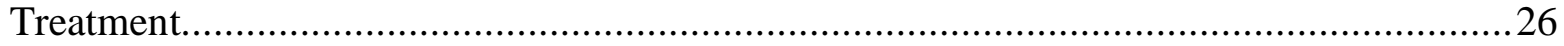

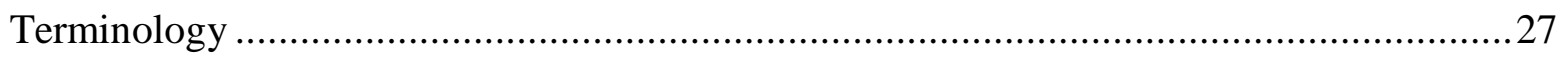

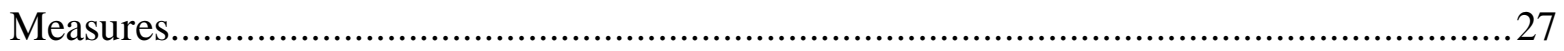

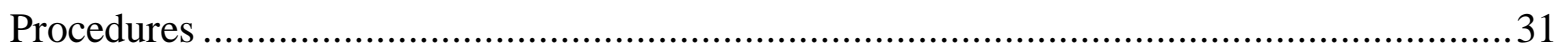

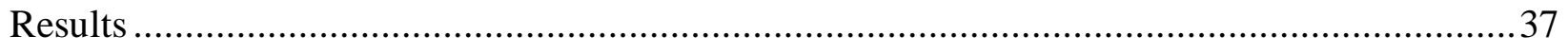

Description of Sudden Gains............................................................................ 37

Impact of the Sudden Gains at the End of Treatment .......................................... 40

Differentiation of Critical Sudden Gain Sessions.....................................................44 
Phenomena after Sudden Gains sessions ........................................................... 46

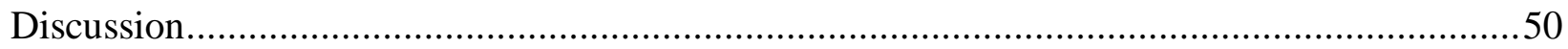

Total CHEDS Sudden Gain............................................................................... 50

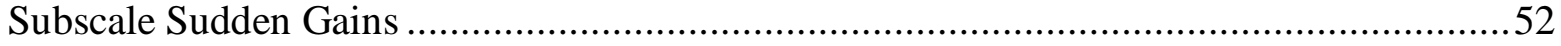

Sudden Gains and the Cognitive Model......................................................................53

Sudden Gains and the Stages of Eating Disorder Treatment .....................................55

Similarity to Other Sudden Gains Findings ............................................................58

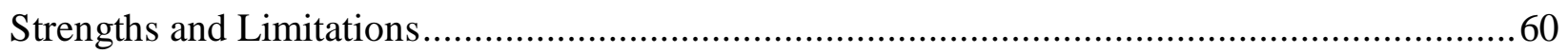

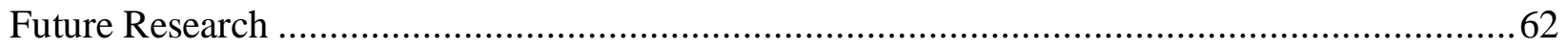

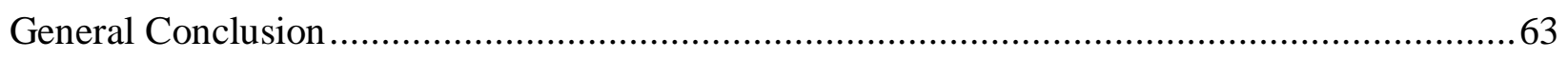

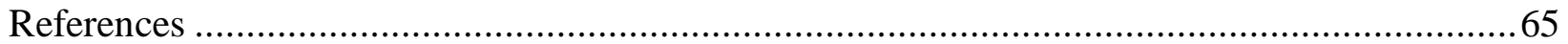




\section{LIST OF TABLES}

Table 1. Relationship Between Proposed Mechanisms and Interventions....................................19

Table 2. Reliability (ICC) for Individual Raters During Training and Rating Process .................31

Table 3. Effect Sizes for Significant Variables after Total CHEDS Sudden Gains .....................47

Table 4. Effect Sizes for Significant Variables after Body Subscale Sudden Gains ....................49 


\section{LIST OF FIGURES}

Figure 1. An illustration of the hypothetical sudden gain trajectory .....................................2

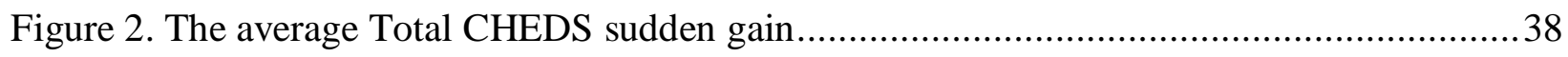

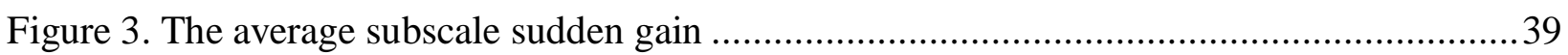

Figure 4. Outcome comparison of clients who experienced Total CHEDS sudden gains and those who did not experience sudden gains

Figure 5. Outcome comparison of clients who experienced Eat subscale and Body subscale sudden gains compared with those who did not experience sudden gains.....

Figure 6. The synchronized time courses of client homework, client cognitive changes and severity of eating disorder symptomatology

Figure 7. The synchronized time courses of client's cognitive changes, severity of eating disorder symptomatology, and behavioral changes... 


\section{Sudden Gains in Cognitive-Behavioral Therapy for Eating Disorders}

\section{Sudden Gains in Cognitive-Behavioral Therapy for Depression}

When examining clients' individual temporal patterns of recovery from depression while receiving cognitive-behavioral therapy (CBT), Tang and colleagues (1999) observed that, in contradiction to the commonly held conception that clients improve gradually, many clients improved suddenly. They noticed that many depressed clients exhibited a dramatic drop in symptomatology between two sessions that remained stable over time resulting in a modified, zshaped recovery pattern. They named this marked symptom improvement pattern "sudden gain".

The existence of sudden gains has been replicated in two variations of CBT (Tang, DeRubeis, Beberman, \& Pham, 2005), and has been found in behavioral activation treatment for depression (Andrusyna, Luborsky, Pham, \& Tang, 2006). CBT sudden gains have been replicated by other investigators (Vittengl, Clark, \& Jarrett, 2005) who have reported sudden gains in depressed samples similar in magnitude and frequency to those reported by Tang and colleagues. Sudden gains have also been found to generalize to naturalistic clinical settings (Hardy et al., 2005) and in pill placebo and pharmacotherapy for depression (Vittengl et al., 2005), indicating the possible generality of sudden gains.

Effects on recovery pattern. Sudden gains appear to account for over $50 \%$ of clients' gains during treatment and to predict lower levels of depression at both post-treatment and at 18 month follow up (Tang \& DeRubeis, 1999b). The effects of sudden gains on long-term outcomes also include better psychosocial functioning (Vittengl et al., 2005) and relapse prevention, with only one third of clients who experienced sudden gains relapsing during the two years following treatment, and showing $74 \%$ less relapse risk than clients who did not experience sudden gains (Andrusyna, Luborsky, Pham, \& Tang, 2006; Tang, DeRubeis, Hollon, 
Amsterdam, \& Shelton, 2007). These long-term outcome findings suggest that sudden gains are enduring improvements in clients' symptoms and not merely symptom fluctuations. As illustrated in Figure 1, a sudden gain refers to a dramatic drop in client reported symptom severity that happens between two sessions and that positively impacts treatment outcome.

Because clients who experience sudden gains have better short- and long-term outcomes, sudden gains findings have received increasing attention in psychotherapy outcome research. Sessions that occur just prior to the sudden gain are considered particularly noteworthy since these sessions are assumed to influence long lasting symptom improvement. Additionally, determining what triggers sudden gains may contribute significantly to the research on mechanisms of change in CBT.

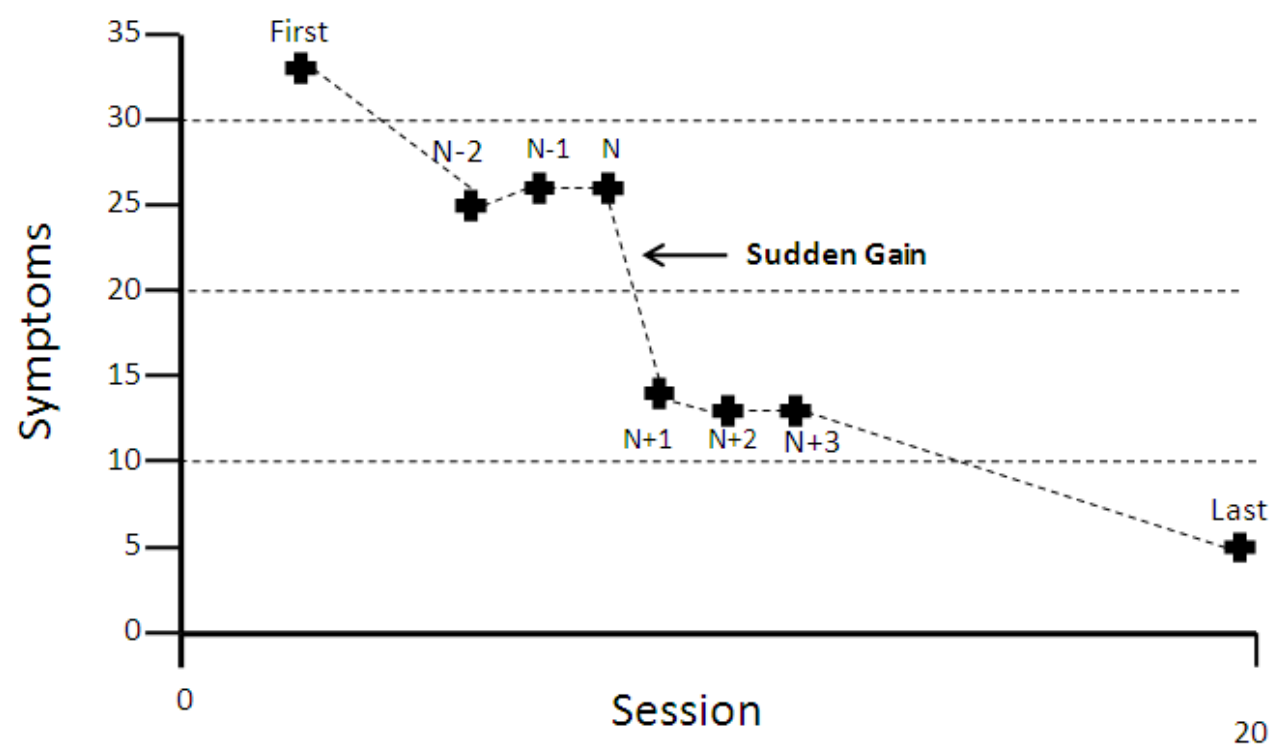

Figure 1. An illustration of the hypothetical sudden gain trajectory, demonstrating what the symptom change would look like for sessions $\mathrm{N}-2, \mathrm{~N}-1, \mathrm{~N}, \mathrm{~N}+1, \mathrm{~N}+2, \mathrm{~N}+3$, with session $\mathrm{N}$ as the pre-gain session. 
Predictors of sudden gains. Predictors of sudden gains identified in previous research include cognitive changes, behavioral changes, and other factors such as client characteristics and the therapeutic alliance.

Cognitive changes. In the original sudden gains studies, the examination of critical sessions that precede the sudden gains explored potential sudden gain predictors such as: clients' cognitive changes (including schema changes, belief changes, and the learning of new cognitive techniques), therapists' application of concrete CBT techniques (i.e., therapists use CBT techniques in an attempt to change clients' beliefs), therapists' application of abstract CBT techniques (i.e., theoretical aspects of CBT, where the therapist and client discuss the cognitive theory of depression and the cognitive mediation hypothesis of CBT and explore client belief systems), and the therapeutic alliance between the therapist and the client (i.e., the extent of the collaborative relationship formed). Of the four, only client cognitive change was significantly different in the critical versus control sessions, in that a substantial amount of cognitive change was observed in the pregain sessions and little cognitive change was observed in the control sessions (Tang \& DeRubeis, 1999b). Additionally, cognitive changes took place in the therapy sessions immediately after the sudden gain occurred. This finding has been replicated (Tang, DeRubeis, Beberman, \& Pham, 2005) and supports the cognitive mediation hypothesis central to CBT, suggesting that cognitive change in the pre-gain sessions contributed to the sudden gains.

These results not only support the cognitive mediation hypothesis, but also suggest a three-stage model for sudden gain recovery where cognitive changes during a critical session lead to sudden gains in symptom reduction, which in turn leads into an upward spiral that includes further cognitive changes, alliance improvement, and greater long-term symptom improvement. This three stage model evident in Tang et al.'s findings was replicated in 
Jacobson's et al. (1996) two variations of CBT, confirming that the CBT sudden gains were preceded by significant cognitive changes in the pre-gain sessions and providing further support to the finding that cognitive changes precede sudden gains (Tang, DeRubeis, Beberman, \& Pham, 2005).

Behavioral changes. Other than cognitive changes, behavioral changes have also been found to predict sudden gains when the sudden gains occurred early in therapy. This finding was expected because behavioral strategies are usually introduced in CBT earlier than cognitive strategies, even though both strategies co-occur for the most part of treatment (Pham, Tang, \& Andrusyna, unpublished). Along similar lines, participants' perspectives on sudden gains indicate that intentions of behavior change are usually carried out by clients and that such behavioral changes influence the development of sudden gains (Pham, 2006). Also, mechanisms of behavioral activation, which include the client's within-session agreement to behavior change and the number of positive activities completed between sessions, have been found to predict sudden gains (Andrusyna, Luborsky, Pham, \& Tang, 2006). These findings suggest that there may be both behavioral and cognitive factors contributing to the occurrence of sudden gains.

Other therapeutic factors. Some factors other than cognitive and behavioral factors have also been investigated as predictors of sudden gains. Client characteristics (e.g., symptom severity, the presence of a comorbid personality disorder, or the clients' age) were not found to be associated with sudden gains, and sudden gains did not appear to reflect positive events in the clients' lives, which provides indirect support for the hypothesis that sudden gains are attributable to factors within the therapy itself rather than to external events that affect clients' lives (Hardy et al., 2005). Critical pre-gain sessions and control sessions have also not been found to differ in so-called common therapeutic factors such as therapeutic alliance; rather, an 
improvement in therapeutic alliance has been found in the sessions immediately after the sudden gains suggesting that therapeutic alliance is a result, not a precursor, of symptom improvement and that additional cognitive change occurs after sudden gains (Tang \& DeRubeis, 1999b). In summary, the literature supports both cognitive and behavioral components of CBT as possible pathways to sudden gains.

\section{Sudden Gains in Other Therapies for Depression}

The literature suggests that sudden gains are not confined to CBT, but are also found in other kinds of therapy for depression. Tang, Luborsky, and Andrusyna (2002) found that clients also show sudden gains in supportive-expressive therapy for depression, with sudden gains being comparable to sudden gains in CBT in terms of the percentage of clients affected and the point in time when they occurred. Sudden gains have been found in psychotherapy for depressed adolescents, including cognitive therapy, systemic behavioral family therapy, and nondirective supportive therapy, being associated with improved outcome on self-report and interviewer ratings of depression (Gaynor et al., 2003). There has also been documentation of sudden gains in interpersonal psychotherapy for depression among women (Kelly, Cyranowski, \& Frank, 2007) and in behavioral activation treatment for depression (Andrusyna, 2008).

However, sudden gains in other forms of therapy are often not as stable as those found in CBT and may well be operating through different mechanisms. Sudden gains in other therapies for depression were much less stable than the CBT sudden gains as indicated by a much higher

rate of symptom reversal before treatment ended, lessened prediction of long-term outcome, and no difference in the likelihood to maintain recovery through maintenance treatment when clients who experienced sudden gains were compared to clients who did not experience sudden gains (Tang, Luborsky, \& Andrusyna, 2002; Kelly et al., 2007). Also, it appears that the mechanisms 
specific to other kinds of therapies were found to be correlated with sudden gains for each of those therapies, and that the observed lack of sudden gains impact on outcome are potentially due to different emphases and diverse mechanisms of change that operate in other therapies.

\section{Sudden Gains in Therapies for Other Diagnoses}

Sudden gains may also be a general phenomenon across treatment for disorders other than depression. Initial findings for disorders other than depression appear to be similar to the sudden gains in therapies for depression, but similar to the findings about sudden gains in other forms of therapy for depression, the sudden gains in therapies for other diagnoses are often not as stable over time. Stiles et al. (2003) conducted the initial investigation of sudden gains outside of depression. They examined a variety of disorders that were treated by a variety of theoretical approaches in a routine clinical setting. Clients' therapies varied in length from seven to 74 individual sessions and those who experienced sudden gains within the first 16 sessions showed significantly better outcome scores when using the short forms of the Clinical Outcomes in Routine Evaluation (CORE-SF; Barkham et al., 2001) as a session-by-session outcome tracking measure. A lower proportion of clients were found to experience sudden gains when compared to those reported by Tang and DeRubeis (1999), but for the clients who did experience sudden gains, their reported symptom change between the sudden gain session interval was as large as the total gain experienced by such clients across their entire treatment. Additionally, since sudden gain contributes to total treatment gain, these clients were more likely to show greater overall gains.

A follow up project on the Stiles et al. (2003) study found that therapists could retrospectively identify which clients had or had not experienced sudden gains as measured by the CORE-SF. Therapists were not familiar with the concept of sudden gains when they 
conducted the therapy and they made their judgments several months to several years after the event, and were still able to identify such clients at rates substantially better than chance rates, which supports the convergent validity of the sudden gains concept (Davies et al., 2006). The findings of Stiles et al (2003) indicate that therapists are partially sensitive to their clients' sudden gain experience, and that therapists' perceptions of sudden gains and its predictors might help them to incorporate knowledge of sudden gains (or lack thereof) into their practice in a way that directly benefits clients.

Panic disorder. Sudden gains have been found in CBT for panic disorder delivered in routine clinical settings (Pham, 2006). The sudden gains in this study of panic disorder were similar to those shown in depression (Tang \& DeRubeis, 1999) in terms of total symptom reduction accounted for, better outcomes at the end of treatment, and overall recovery, although less stable in matters of symptom reversal. An additional study of sudden gains in CBT for panic disorder concluded that sudden gains occurring after session two predicted overall symptom reduction at the end of treatment and some changes in cognitive biases (Clerkin, Teachman, \& Smith-Janik, 2008).

Other anxiety disorders. Sudden gains have also been reported in 107 participants with social anxiety disorder who received either group CBT or exposure group therapy without explicit cognitive interventions. Clients with sudden gains showed similar improvements in both treatment groups and even though CBT was associated with more cognitive changes than exposure therapy, cognitive changes did not always precede sudden gains (Hofmann, Schulz, Meuret, Moscovitch, \& Suvak, 2006). Sudden gains have also been noted in brief supportiveexpressive psychotherapy for generalized anxiety disorder. However, in this form of therapy 
many clients experienced reversals of these gains and lost over $50 \%$ of the sudden gain during subsequent treatment sessions (Present et al., 2008).

Overall, sudden gains appear to be a general phenomenon occurring in different kinds of therapies for several types of disorders. Sudden gains are not specific to mood changes seen in depression; rather, sudden gains appear to represent genuine symptom reduction. However, in therapies other than CBT for depression, sudden gains have been found to be more unstable in the long-term as shown by patterns of reversal of symptoms and less predictability of long-term outcome. Furthermore, initial studies also suggest that the mechanisms of sudden gains may differ across therapies and diagnostic groups.

\section{Other Recovery Patterns}

Early change versus sudden gain. In studies of therapeutic mechanisms through the examination of time course data, a concept related to sudden gains has emerged. What is termed early or rapid change has been identified as therapeutic gains that take place during the first four weeks (usually first 6-8 sessions) of CBT, which often accounts for 60-70\% of overall symptom improvement during CBT for depression (Ilardi \& Craighead, 1994). Early change varies between clients and can be minimal or substantial. An early change is different from a sudden gain because it includes only therapeutic gains that occur during the first four weeks (or first 6-8 sessions) of treatment. By contrast, sudden gains refer exclusively to significant symptom reduction that occurs between any two sessions at any point in treatment and remains stable for a specific time, thereafter resulting in a z-shaped recovery pattern as previously depicted in Figure 1. Nonetheless, predictors of early change may hold implications for potential predictors of sudden gains. 
Predictors of early change. Initially, early change was attributed to common therapeutic factors (such as hope, alliance, etc) because it was assumed that cognitive modification techniques were not extensively applied during the first few weeks of treatment, leading to the idea that Beck's cognitive mediation hypothesis of CBT would not be able to explain the early change (Ilardi \& Craighead, 1994). However, it has been observed that cognitive modification techniques are applied extensively in early sessions, especially because during the first four weeks of CBT session are typically held twice weekly, allowing for eight sessions during which cognitive modification techniques are consistently applied (Tang \& DeRubeis, 1999a).

When the time and effort spent by CBT therapists on cognitive modification was measured in four therapy sessions randomly selected, it was concluded that CBT therapists spent the same amount of time and effort working on cognitive interventions in the second session as they did in later sessions (Feeley \& DeRubeis, 1999), suggesting that CBT therapists spend substantial time applying cognitive modification techniques in early CBT sessions. It has been further pointed out that because $40-60 \%$ of therapy sessions take place in the first 4 weeks of treatment, it makes sense that cognitive techniques would be so forcefully applied, indicating that the available time course data does not contradict Beck's cognitive mediation hypothesis of CBT even though it does not directly support it either (Tang \& DeRubeis, 1999a).

Generality of early change. The phenomenon of early change response to CBT appears to be a more general phenomenon, applying to the treatment of disorders other than depression. Wilson (1999a) described how early change has been found in CBT for alcohol abuse, panic disorder, and bulimia nervosa. The work on early gain in bulimia nervosa has been replicated and expanded by Fairburn, Agras, Walsh, Wilson, and Stice (2004). In a sample of 220 women treated with either CBT or interpersonal psychotherapy, early change in frequency of purging 
was the best predictor of outcome, both at the end of treatment and at 8-month follow-up. Early change in treatment then appears to directly apply to eating disorders and to be a strong and potent predictor of immediate and longer-term outcome in CBT for bulimia nervosa, suggesting that the first few weeks of treatment are important and that effort should be made to increase early behavior change.

The studies on early change have led to recommendations to study session-by-session time course data when investigating change patterns and/or purported mechanisms of change. Early change and sudden gain are different concepts, both of which were found to exist through the examination of time course data. Although the currently proposed study focuses on sudden gains exclusively, the research on early change has implications for understanding recovery patterns and mechanisms of change occurring during therapy, and is thus relevant for identification of potential predictors of sudden gains as a distinct recovery trajectory pattern found in CBT.

\section{Cognitive-Behavioral Therapy for Eating Disorders}

Given what has been found regarding sudden gains in CBT for depression and its extension to other forms of therapy and other disorders, sudden gains may also be operative in CBT for eating disorders. Although no study of sudden gains in eating disorder treatment has as yet been published, exploring the potential existence of sudden gains in those with eating disorders may produce valuable knowledge that could impact current treatment methods.

Since Beck and colleagues published the first CBT treatment manual in 1979, there has been increased evidence regarding the efficacy of CBT for a number of disorders. CBT was originally developed for depression, and the efficacy of CBT for depression has been well established (Blackburn, Bishop, Glen, Whalley, \& Christie, 1981; DeRubeis, Gelfand, Tang, \& 
Simons, 1999). CBT has been modified for use with many other disorders and subsequently been found to be effective in the treatment of many other disorders such as generalized anxiety disorder, panic disorder, social anxiety, post-traumatic stress disorder, obsessive compulsive disorder, borderline personality disorder, sexual dysfunctions, schizophrenia and bipolar disorder (Barlow, 2001; Butler, Chapman, Forman, \& Beck, 2006). Such expansion of scope happened as researchers applied and modified the general principles of CBT to specific diagnostic groups. With respect to eating disorders, a specific CBT treatment protocol for eating disorders has been developed and has been strongly supported as an effective treatment for eating disorders. Fairburn first developed this eating disorder specific treatment in 1981 followed by revisions of the protocol in 1993 and 2008 (Fairburn, 1981, 2008; Fairburn, Marcus, \& Wilson, 1993). This CBT treatment for eating disorders focuses on increasing regularity of eating, eliminating food avoidance, and changing maladaptive attitudes toward food, eating and the body. There is now convincing evidence that CBT is broadly applicable to, and effective in, the treatment of the entire spectrum of eating disorders (Fairburn, 1995, 2009; Lewandowski, Gebing, Anthony, \& O'Brien, 1997; Whittal \& Agras, 1999; Wilson \& Fairburn, 1993, 1998). CBT has also been recommended as the treatment of choice for bulimia nervosa and binge eating disorder by several expert task forces in several countries (e.g Fairburn, Agras, \& Wilson, 1992). Pike, Devlin, \& Loeb (2004) have articulated a set of assumptions and principles that define and describe CBT for the treatment of the eating disorders and reviewed the empirical support of CBT in the treatment of anorexia nervosa, bulimia nervosa, and binge eating disorder, and concluded, like other investigators, that CBT is effective in treating such disorders and that therapists providing treatment for eating disorders should be familiar with the empirically supported CBT treatment protocols. 
In the most recent iteration of CBT treatment for eating disorders, Fairburn (2008) developed an enhanced CBT protocol for eating disorders to address specific eating disorder psychopathology, which can be employed as a core treatment, or as a broader version including modules that address clinical perfectionism, low self-esteem, mood intolerance, and interpersonal difficulties which often co-occur with eating disorders. This treatment focuses on eating disorders as being maintained by dysfunctional beliefs about the body and about food that lead to dissatisfaction with shape and weight, which then leads to the development of rigid eating patterns aimed at controlling and changing shape and weight. The core treatment consists of 20 individual sessions conducted over 20 weeks. Each session occurs twice a week for the first three weeks, weekly for the next 12 weeks, and then every other week for the last six weeks.

The protocol is divided into four stages, each focusing on specific eating disorder symptomatology. Stage One is aimed at reducing and eventually eliminating excessive dietary restriction, binge eating, and compensatory behaviors. In this stage there are many behavioral goals worked on to regularize the client's chaotic eating and much information conveyed regarding the effects of dietary restriction, ineffectiveness of vomiting and laxative use, physical consequences of binge eating and purging, and determining an individual's healthy weight range. Stage Two is aimed at assessing changes made in Stage One, identifying any barriers for change, and planning Stage Three. Stage Three focuses on modifying client dysfunctional beliefs about eating and about body shape and weight that contribute to body dissatisfaction and dietary restriction, addressing what types of foods are eaten, how much food is eaten, and body image. Stage Three also aims at increasing problem solving abilities to decrease the use of eating as a mood regulator, allowing the client to have more alternatives when coping with stressful 
situations. Stage Four focuses on maintenance of treatment gains and relapse prevention (Fairburn, 2008; Spangler, 1999).

Given that CBT was first developed for depression and later expanded and modified to treat eating disorders specifically, and the recent findings regarding sudden gains in CBT for depression and other disorders, it appears possible that sudden gains may be present in CBT for eating disorders and that such investigation may contribute to the understanding of recovery patterns and recovery mechanisms in CBT for eating disorders.

\section{Predictors of Sudden Gains in CBT for Eating Disorders}

If sudden gains are present during CBT for eating disorders, then examining predictors of sudden gains could aid in understanding the processes through which sudden gains in eating disordered symptoms occur. There are different aspects of treatment that are important to be distinguished when considering what may contribute to the occurrence of sudden gains. Within the CBT approach to the treatment of eating disorders described above, therapist interventions and treatment mechanisms are differentiated as aspects of treatment that interact and affect symptom change. Both therapist intervention and client mechanisms are of interest when studying how treatment proceeds in the direction of the desired outcome.

Therapist interventions refer to a treatment manipulation introduced by the therapist as a catalyst for client change. In other words, therapist interventions are therapeutic acts performed by the therapist within the therapy session with the intent of modifying the outcome. In CBT for eating disorders therapist interventions include behavioral interventions, cognitive interventions, structural interventions, and the interpersonal style in which such activities are delivered (Hollon \& Kriss, 1984; Spangler, Baldwin \& Agras, 2004). Treatment mechanisms are different than therapist interventions because mechanisms refer to the clients' characteristics that change 
during treatment and directly produce symptom reduction. While interventions are initiated and conducted by the therapist, mechanisms are exclusive to clients. In CBT for eating disorders, client mechanisms include variables such as dietary restraint and beliefs about the body. Eating disorder symptoms are also pertinent to the client, but client mechanisms are theoretically differentiated from symptoms because of their temporal precedence and effect on symptoms in the treatment of eating disorders.

A model has been proposed to explain the connection between therapist interventions, treatment mechanisms, and eating disorder symptoms. The model indicates that therapist interventions directly affect client mechanisms, which in turn, influence symptom change (Hollon \& Kriss, 1984; Spangler, Baldwin, \& Agras, 2004). This model indicates that therapist interventions are actions done by the therapist with the intention of creating outcome change; such interventions influence change in specific client mechanisms; the change in mechanisms then is responsible for the change in client symptoms. Even though client mechanisms are similar to symptoms because they are clients' characteristics that change during treatment, mechanisms are theoretically different from symptoms because symptom change depends on the change of specific mechanisms. For example, a client's eating behavior is one mechanism that would need to change (from dysregulated eating to regular eating) in order for the symptom of binge eating to change. Engagement in regular eating is in this case the mechanism responsible for changes in binge eating. Mechanisms of change are thus proposed to mediate the relationship between therapist interventions and symptom change. In order to establish how a treatment works, both client mechanisms and therapist manipulations are usually investigated; however, because client mechanisms have been identified as mediating the relationship between therapist 
interventions and symptom change, client mechanisms may be found to be more prominent among predictors of sudden gains.

Eating disorder symptoms. The symptoms that characterize eating disorders are expected to change through the interaction between therapist interventions and client mechanisms as explained above (Spangler et al., 2004). Such symptoms include intense fear of gaining weight or becoming fat, disturbance in the way in which one's body weight or shape is experienced, body dissatisfaction, difficulty focusing on other things due to concern with body shape and weight, a strong desire to change the body, episodes of binge eating (rapidly eating large amounts of food accompanied by a sensation of loss of control), inappropriate compensatory behaviors (self-induced vomiting, misuse of laxatives, diuretics, or other medications, fasting, or excessive exercise), trying to restrict food intake, preoccupation with thoughts about food, and fear of eating due to possible loss of control (APA, 1994).

The symptoms described above are easily divided into two categories that compose the major domains of the eating disorder spectrum. The first category includes eating related thoughts, feeling, and behaviors (e.g. feeling like one cannot stop eating, eating more rapidly than normal, restricting the amount of food eaten, vomiting, etc); the second category includes body related thoughts, feelings, and behaviors (e.g. sense of self worth influenced by the body shape and weight, desire to change the body, desire to become thinner, etc.). The indication that there are two primary, distinct domains in the symptoms of eating disorders point to the idea that therapist interventions are designed to address both domains of symptoms, but that different mechanisms may mediate the relationship between therapist interventions and the two different domains of symptoms. 
Therapist interventions. As explained in the Fairburn protocol for CBT for eating disorders (Fairburn, 1981, 2008; Fairburn et al., 1993), there are several treatment interventions that are thought to influence symptom change. The interventions introduced by the therapist within the therapy session with the intent of modifying the outcome fall within the different domains of psychoeducation, behavioral interventions, cognitive interventions, relational interventions, and structural interventions. All of these are thought to contribute to change in specific client mechanism variables and to symptom change.

Psychoeducation is intended to inform the client about the eating problem and the myths about eating disorders and weight control. More specifically, clients are informed about their eating disorder diagnosis, prevalences and characteristics, associated health risks, treatment and prognosis, characteristic extreme concerns about shape and weight, characteristic forms of dieting, effects of dietary restriction, binge eating and its consequences, effects of self-induced vomiting, effects of laxative and diuretic misuse, concerns with over-exercising, and consequences of being underweight (Fairburn, 1995, 2008).

Behavioral interventions are intended to promote change mechanisms that create change in eating and food related symptoms such as dietary restriction, binge eating, and self-induced vomiting. Behavioral interventions present in $\mathrm{CBT}$ for eating disorders include prescription of regular eating, meal planning, exposure to feared foods, food and body related experiments, evaluation of specific behaviors, exploration and evaluation of behaviors, planning and practicing alternative behaviors, skills training, scheduling and structuring activities, and manipulating behaviors via cues or consequences (Fairburn, 2008; Spangler et al., 2001). Psychoeducation and behavioral interventions are therapist interventions predicted to contribute to changes in client mechanisms such as dietary restriction and eating behaviors during 
treatment, and thereby reduce eating/food related symptoms such as binge eating, food preoccupation, feeling like one cannot stop eating, eating more rapidly than normal, food restricting, and vomiting (Spangler et al., 2004).

Cognitive interventions are intended to primarily assist in changing body related symptoms such as difficulty focusing on other things due to thoughts about the body, fear of weight gain, desire to lose weight, and self-worth unduly influenced by shape and weight. Cognitive interventions present in CBT for eating disorders include monitoring of cognitions, focusing on key cognitions while exploring relationship of thoughts and feelings, exploring of developing underlying assumptions, exploring personal meaning, evaluating and changing cognition by recognizing cognitive errors, distancing beliefs, examining available evidence, testing beliefs, searching for alternative explanations, exploring the adaptive value of a belief, and practicing rational responses (Fairburn, 2008; Spangler et al., 2001). Monitoring of cognition and therapist interventions designed to explore and modify cognition, as well as bodyrelated behavioral experiments and psychoeducation, are thought to bring about change in client mechanisms of body related thoughts, feelings, and behaviors; such as client beliefs about and investment in body appearance during treatment (Fairburn, 2008; Spangler et al., 2004).

Relational interventions include the quality of delivery and the therapist interpersonal style in which the interventions are delivered (Hollon \& Kriss, 1984). Relational interventions include empathy, warmth, rapport, understanding, interpersonal effectiveness, and collaboration and involvement between client and therapist. Structural interventions include the therapist structuring of sessions with the intent of making therapy more understandable for the client, to help clients understand their responsibilities, increase client engagement and choice, and to increase the likelihood that clients will be able to retain gains after treatment termination (Beck, 
1995). Structural interventions include agenda setting, pacing and efficient use of time, setting and reviewing homework, and providing a rationale for specific interventions (Spangler et al., 2001). Relational and structural interventions have been found to have an impact on client engagement (e.g. client participation in session, client involvement in treatment) and to also increase changes in eating and body related eating disorder symptoms (Spangler et al., 2004).

Mechanisms of change. The therapist interventions or treatment manipulations mentioned above are believed to directly affect client change during treatment and therefore to indirectly produce symptom reduction. Wilson and Fairburn (1993) mentioned a few client mechanisms specific to eating disorders that directly influence symptom change: abnormal attitudes toward body shape and weight, dietary restraint, self-efficacy and coping (for eating a larger amount of more diverse foods without losing control and for coping with situations that trigger binge eating), negative reinforcement received from purging (when binge eating is no longer inhibited by anxiety about weight gain) and association between external cues and binge eating (triggers to binge eating). According to CBT theory and practice, mechanisms including change in client dysfunctional beliefs about appearance, engagement in regular eating (changes in dietary restriction), and client engagement in treatment have been proposed as important mechanisms of action in CBT for eating disorders (Spangler et al., 2004).

The work of Spangler and colleagues (2004) in investigating the relationships between therapist interventions, client mechanisms and symptom change indicated that increases in behavioral interventions were significantly associated with decreases in dietary restriction, and decreases in weight and shape concern. Additionally, decreases in behavioral interventions were associated with increased change in body-related dysfunctional beliefs during treatment. Such findings confirm that increased utilization of specific interventions enhance the change of 
Table 1

Relationship BetweenProposed Interventions and Mechanisms

\begin{tabular}{|c|c|c|c|c|}
\hline Interventions & $\rightarrow$ & Mechanisms & $\rightarrow$ & Symptoms \\
\hline Behavioral Interventions & & Regular Eating & & $\begin{array}{l}\text { Binge Eating } \\
\text { Vomiting } \\
\text { Food/Eating Preoccupation } \\
\text { Dietary Restriction }\end{array}$ \\
\hline Cognitive Interventions & & Body Schema & & $\begin{array}{l}\text { Body Dissatisfaction } \\
\text { Body Preocupation }\end{array}$ \\
\hline Relational Interventions & & Client Engagement & & \\
\hline Structural Interventions & & Client Engagement & & \\
\hline
\end{tabular}

specific mechanisms, which in turn create the desired symptomatology change. The relationship of client mechanisms as a mediator between therapist interventions and eating disorder symptom change is exemplified in Table 1.

Engagement in regular eating. Client change in regularity of eating behaviors has been examined as an important treatment mechanism of CBT for eating disorders. CBT for eating disorders has been shown to have a significantly more rapid time course in decreasing binge eating and purging than alternative forms of treatment such as interpersonal psychotherapy (Agras et al., 2000; Fairburn, Jones, Peveler, \& Carr, 1991; Jones, Peveler, \& Hope, 1993), supportive-expressive therapy (Garner, Rockert, Davis, \& Garner, 1993), and psychodynamic therapy (Walsh, Wilson, Loeb, \& Devlin, 1997; Wilson, 1999b). Part of this research has focused on the mechanisms of early change (Agras et al., 2000; Wilson, Fairburn, Agras, Walsh, \& Kraemer, 2002), indicating that reduction of purging by session six differentiates clients who do and do not respond to cognitive behavior therapy for bulimia nervosa, and that rapid change in purging predicts better acute and long-term outcomes. In addition, greater dietary restriction 
was found to characterize participants with treatment failures. Such predictors are relevant to the current project because even though they were originally identified as predictors of early change, it is assumed that the same client mechanisms possibly apply to the prediction of sudden gains in CBT for eating disorders.

In the research that has specifically examined mechanisms of change in CBT for eating disorders, engagement in regular eating was found to be associated with decreased vomiting frequency and decreased weight concern, while increases in dietary restraint predicted increases in weight concerns and in vomiting. It has been further specified that as clients eat more regularly, they binge eat less often, and consequently experience a decrease in purging, which is often experienced as a consequence of perceived binge eating (Spangler et al., 2004), supporting the hypotheses of theorists (e.g., Fairburn, 1981, 1997) who propose that dietary restriction leads to binge eating and purging.

Beliefs about shape and weight. CBT theory indicates that as dysfunctional beliefs about the importance of body appearance decrease, the desire for thinness, negative automatic thoughts about the body, concern about body shape and weight, and dietary restriction consequently decrease (Spangler et al., 2004). In support of this hypothesis, CBT produces greater change in client cognitive variables such as body image, eating attitudes, and attitudes toward body shape and weight than other forms of treatment or purely behavioral versions of CBT (Fairburn et al., 1991; Garner et al., 1993; Jones et al., 1993; Rosen, 1996; Thackwray \& Smith, 1993; Whittal \& Agras, 1999). Increased change in body-related dysfunctional beliefs has been found to be significantly associated with decreases in weight concern during CBT treatment for eating disorders, which supports the theory that change in client body-related 
dysfunctional beliefs leads to change in eating disorder symptoms such as level of concern about the body (Spangler et al., 2004).

Client engagement. The mechanism of client engagement, which refers to the active participation and involvement in the treatment process, has also been found to be related to vomiting frequency, where, according to prediction, increased client engagement in treatment was associated with decreased vomiting (Spangler et al., 2004), which supports the purported importance of client motivation, hope, and involvement in treatment for change to occur as hypothesized by both CBT (e.g., Burns, 1989; Leahy, 2001) and common factor (Grencavage \& Norcross, 1990; Wampold, 2001) theorists.

Client engagement in homework assignments (homework commitment and homework completion) is one important aspect of client engagement in CBT that may predict sudden gains. Homework in CBT for eating disorders may include a variety of behavioral and cognitive tasks such as monitoring eating, planning meals, problem solving for binge eating and restriction cues, decreasing body shape checking, and both monitoring and challenging of thoughts related to feeling fat (Fairburn, 1995, 2008). The causal relationship between psychotherapy homework compliance and changes in symptoms has been analyzed and results support the hypothesis that homework compliance has a causal effect on changes in depression, where clients who did the most homework improved much more (Burns \& Spangler, 2000). Behavioral homework assignments have also been proposed as a promising candidate for explaining the rapid change found in CBT because CBT is unique in regards to the systematic use of behavioral homework assignments (Wilson, 1999a). Again, early change is a different concept when compared to sudden gains, but its mechanisms of action could be shared by the phenomenon of sudden gains. 
Conclusion. It is possible that predictors of sudden gains for eating disorders will include some of the predictors mentioned in the previous research done in sudden gains because there are many cognitive and behavioral components of CBT that are common to the treatment of any specific disorder. The general CBT aspects applied to eating disorders include relational interventions (e.g. therapist empathy), psychoeducation, cognitive interventions, behavioral interventions, and structural interventions (e.g. homework assignment, agenda setting) (Spangler et al., 2001). In addition, it is also important examine how such general CBT principles apply to the specific psychopathology of eating disorders and how they interact with treatment mechanisms because the general components of CBT may be predictors of sudden gains only when specifically applied to the more particular dysfunctional cognitions and behaviors seen in eating disorders. It is also possible that predictors previously examined in early gain research will be found to be predictors of sudden gain because the same mechanisms of action could be applicable to different patterns of recovery.

The research on sudden gains and on early gains and the research on treatment mechanisms in CBT for eating disorders indicate that behavioral and cognitive changes through in-session application of therapist interventions or through homework could be predictors of sudden gains in CBT for eating disorders. Such mechanisms of action are hypothesized to be present in critical sessions and to possibly differentiate critical sessions from other therapy sessions, but the focus of this study is not to identify mechanisms of change but to identify what precedes the major symptom shift of sudden gains. A broad range of factors will be investigated as possible predictors of sudden gains, including therapist interventions (e.g. agenda setting, exploration of behavior and cognition, and evaluation and change of behavior and cognition), 
client changes (e.g. changes in beliefs about shape and weight, changes in eating related behaviors, etc), engagement in homework assignments, and the therapeutic relationship.

\section{Study Aims and Hypotheses}

The purpose of the present study was to evaluate the existence of sudden gains in CBT for eating disorders and to explore if pre-gain sessions can be differentiated from other therapy sessions. More specifically, research questions addressed in this study included:

1. Is the phenomenon of sudden gains present in CBT for eating disorders?

2. If present, do sudden gains in CBT for eating disorders follow the three-stage model for sudden gain recovery where cognitive changes during a critical session lead to sudden gains, which in turn lead into an upward spiral that includes further cognitive changes, alliance improvement, and greater long-term symptom improvement?

3. If present, what are the predictors of sudden gains that distinguish the critical session that takes place right before the sudden gain from other therapy sessions?

It was hypothesized that:

1. Sudden gains similar to the ones previously reported for other disorders (see Figure 1) are present in CBT for eating disorders, with sudden gains occurring in more than $50 \%$ of the CBT responders, accounting for more than $50 \%$ of these clients total symptom improvements, with no more than $17 \%$ of sudden gainers experiencing reversal before the end of therapy, with $50 \%$ of sudden gains taking place between sessions 4 and 10, and with acute outcomes being significantly better for sudden gainers.

2. The pregain session will differ from other therapy sessions in matters of therapist structural, cognitive, behavioral, and relational interventions, client cognitive (beliefs 
about shape and weight) and behavioral changes (engagement in regular eating), client engagement in session, and engagement in homework assignments, which were hypothesized to be predictors of sudden gains in CBT for eating disorders.

3. Two different kinds of sudden gains in CBT for eating disorders exist, one taking place due to changes in eating related thoughts, feeling, and behaviors (e.g. feeling like one cannot stop eating, eating more rapidly than normal, restricting the amount of food eaten, vomiting, etc) and another due to changes in and body related thoughts, feelings, and behaviors (e.g. body checking, body dissatisfaction, desire to become thinner, etc.).

4. Sudden gains due to changes in eating related thoughts, feeling, and behaviors will be primarily predicted by client behavioral changes (e.g. engagement in regular eating).

5. Sudden gains due to changes in body related thoughts, feelings, and behaviors will be primarily predicted by client cognitive changes (e.g. changes in beliefs about shape).

The present study investigated whether sudden gains are evident in eating disorders and whether they have significant implications for understanding the temporal pattern and predictors of recovery from eating disorders. The existence of sudden gains and an understanding of the characteristics of critical therapy sessions could identify aspects of treatment that CBT therapists may focus on to improve client recovery.

\section{Method}

\section{Data}

Data were obtained from Spangler's (2009) efficacy study of CBT for eating disorders. This study included videotapes of all therapy sessions, as well as an outcome tracking measure (CHEDS) given before every session. 


\section{Participants}

Participants were recruited through fliers, newspaper ads, and referrals to a mental health community outpatient clinic. Potential participants were initially screened by telephone, which was followed by an in-person diagnostic assessment. The Eating Disorder Examination (EDE; Cooper \& Fairburn, 1987; Fairburn \& Cooper, 1993) was used to diagnose clients for admission into the study. The EDE is an interviewer-based measure used to assess the presence of an eating disorder and the severity of eating disorder symptomatology with moderate to excellent interrater reliability (Fairburn \& Cooper, 1993; Wilson \& Smith, 1989) high internal consistency (Cooper, Cooper, \& Fairburn, 1989), and discriminant and concurrent validity (Fairburn \& Cooper, 1993). Assessors were supervised weekly on-site by Dr. Diane Spangler who was trained in the use of the EDE by the originators of the scale.

Participants were excluded from the study if they had comorbid psychosis or bipolar disorder because some medications to treat such conditions may significantly impact weight and because those disorders may impair the thought organization necessary for engagement in psychotherapy. Participants were also excluded if they had a medical condition that significantly impacts weight (e.g., thyroid conditions) or if they had a history of bariatric surgery.

Additionally, participants were not allowed to concurrently participate in any other type of psychotherapeutic treatment, although concurrent medication use was allowed. Participants signed an informed consent for participation in the study, videotaping, and completion of the measures. All participants received therapy at no-cost. Fifty-three participants were included in the current study which specifically excluded clients who met criteria for anorexia nervosa ${ }^{1}$. Of

\footnotetext{
${ }^{1}$ Anorexia nervosa has been excluded from this study because of its uniqueness when compared to the whole of the eating disorders spectrum. Many in the field view anorexia nervosa as a rather distinct group, and the treatment outcomes of those with anorexia nervosa are found to be different from the other eating disorders leading to hypotheses that the mechanisms of action of treatment may be different in anorexia (G. T. Wilson \& Fairburn, 1998). Additionally, given the small number of participants who met full criteria for AN in the current treatment study, a separate analysis of AN participants was not feasible.
} 
these 53 clients, $46 \%$ meet criteria for bulimia nervosa, $29 \%$ met criteria for binge eating disorder and 25\% met criteria for EDNOS. The majority of the participants were female (93\%) and Caucasian (98\%) with 2\% reporting a Hispanic ethnicity. Age ranged from 18 to 65 ( $M=$ 25.24, $S D=9.97)$.

\section{Therapists}

Therapists were four female $\mathrm{PhD}$ candidates in clinical psychology. They all had previously received training in cognitive behavioral theory and interventions, and had been practicing cognitive behavioral therapy under supervision for at least one year. All therapists were trained by Dr. Diane Spangler in the use of the Fairburn eating disorder focused CBT protocol (Fairburn, 2008) prior to beginning treatment with study clients, including seeing pilot clients until competency in delivering the treatment protocol was reached. Therapists received weekly supervision on site with videotaped session review and completed fidelity checks with the treatment protocol at each session.

\section{Treatment}

Treatment was administered on an outpatient basis consisting of 20 individual sessions conducted over 20 weeks. Each session was 50 minutes in length and occurred twice weekly for the first three weeks, weekly for the next 11 weeks, and once every two weeks for the last six weeks of treatment. The protocol used is divided into four stages, each focusing on specific eating disorder symptomatology. Stage One focused on reducing and eventually eliminating excessive dietary restriction, binge eating, and compensatory behaviors. Stage Two focused on assessing changes made in Stage one, identifying any barriers for change, and planning of Stage Three. Stage Three focused on modifying client dysfunctional beliefs about body shape and weight. Stage Four focused on maintenance of treatment gains and relapse prevention. A 
detailed description of the treatment protocol is provided by Fairburn (2008). Each session in this treatment protocol had specified agenda items with assessments and clinical forms to be used. While the treatment protocol is manualized, crucial elements of CBT such as tailoring treatment for specific clients' needs and symptoms were emphasized.

\section{Terminology}

Consistent with the terminology introduced by Tang and DeRubeis (1999) and as depicted in Figure 1, the therapy session immediately preceding the sudden gain is referred to as the pre-gain or critical session and the therapy session immediately after the gain as the postgain session. In addition, the session before the pre-gain session is referred to as the pre-pregain session. Thus, the temporal sequence of sessions is as follows: pre-pregain session $(\mathrm{N}-1) \longrightarrow$ pre-gain session $(\mathrm{N}) \longrightarrow$ sudden gain $\longrightarrow$ post-gain session $(\mathrm{N}+1)$.

\section{Measures}

Change in eating disorder symptomatology. The Change in Eating Disorder Symptoms Scale (CHEDS; Spangler, 2010) is a 35 item, comprehensive measure of eating disorder symptomatology that measures the primary symptoms of eating disorders. Previous studies have demonstrated the reliability and validity of the CHEDS. The factor structure of the CHEDS consists of seven factors including: eating concerns/preoccupation, restriction, body preoccupation, body dissatisfaction, body checking, vomiting, and binge eating (Spangler, 2010). The seven factors accounted for $72 \%$ of the variance. The internal reliabilities of the CHEDS subscales range from .85 to .93 , with the exception of one subscale at .73 , while the overall internal reliability coefficient alpha was .96 . The factor with the lowest internal reliability, vomiting, only contained two items. 
The CHEDS has also demonstrated high reliability, high construct validity (the subscales correlated in expected patterns with other measures), high discriminant validity (being able to discriminate between eating disordered and non-eating disordered groups), and high concurrent validity with other eating disorder measures. The temporal (test-retest) reliability of the CHEDS has been found to be .90 , indicating that, without intervention, eating disorder symptoms tend to be stable over time (Spangler, 2010). CHEDS items are also sensitive to change as the items change in the theoretically proposed direction during treatment (Hwang \& Spangler, in progress).

The CHEDS can be divided into two subscales: eating related thoughts, feelings, and behaviors (e.g. feeling like one cannot stop eating, eating more rapidly than normal, restricting the amount of food eaten, vomiting, etc) and body related thoughts, feelings, and behaviors (e.g. sense of self worth influenced by the body shape and weight, desire to change the body, desire to become thinner, etc.). These two subscales reflect the major domains of symptoms seen in eating disorders, and the separation of such symptoms is relevant to the above mentioned hypothesis number three of this study, where two different kinds of sudden gains in CBT for eating disorders are hypothesized to exist (one taking place due to changes in eating related symptoms and another due to changes in and body related symptoms).

Predictors of sudden gains in critical sessions. The CBT Coding Scale for Bulimia Nervosa (CCS-BN; Spangler et al., 2001) is an observer coded scale composed of 69 items and was used to assess therapist interventions, client changes in the mechanisms of beliefs about shape and weight, restrictive eating, and client engagement in session and homework assignments. The CCS-BN is composed of separate therapist and client sections.

The therapist section includes subscales designed to assess therapist use and quality of execution of structural, behavioral, cognitive, and relational interventions on a Likert scale 
allowing ratings from 0 (not executed) to 6 (very well executed). The structural subscale is composed of two items with an alpha of .99 and assesses the degree to which therapists set and follow an agenda during the session, allowing efficient use of time. The behavioral subscale is composed of six items with an alpha of .80 and assesses the extent to which therapists explore problematic behaviors, plan and practice alternative behaviors during the session, attempt to engage the client in skill training, schedule or structure activities with the client, and help to identify cues and/or consequences for specific undesirable behaviors. The cognitive subscale is composed of 15 items with an alpha of .87 and assesses the extent to which therapists explore cognitions during sessions, and the quality of their use of various techniques for restructuring cognitions such as empiricism, recognizing cognitive errors, examining the evidence, generating alternative explanations, etc. The empathy subscale is composed of seven items with an alpha of .97 and assesses the degree to which therapists demonstrate empathy, warmth, understanding, interpersonal effectiveness, collaboration, and involvement during sessions. The homework subscale is composed of four items with an alpha of .73, and assesses therapists' attempt to incorporate relevant homework, homework assigned, and homework reviewed.

The several therapist subscales or factors, as described above, have been verified using both exploratory and confirmatory factor analyses. The therapist portion of the scale has been shown to possess adequate interrater reliability (Spangler et al., 2001). Interrater correlations for the therapist subscales of cognitive, behavioral, relational, and structural interventions were found to be $.69, .64, .71$, and .79, respectively, with a $p<.01$ for all (Spangler et al., 2004). The therapist section of the CCS-BN will be used to measure therapist structural, cognitive, behavioral, and relational interventions. 
The client portion of the scale assesses client change in eating behaviors and beliefs, in body-related behaviors and dysfunctional beliefs, and the degree of client engagement in a given session using a Likert scale ranging from none (0) to extremely (6). The symptom change subscale has a coefficient alpha of .94 and assesses the degree to which clients attempted to change binge and purge related behaviors and thoughts (i.e. practice new eating behaviors, change food thoughts, change purge thoughts, etc). The body subscale has an alpha of .89 and assesses the clients' attempts to change body related thoughts, and body related behaviors (i.e. body checking, etc). The client engagement subscale has an alpha of .91 and assesses degree of client investment in treatment, including client engagement, client motivation, use of selfmonitoring, and amount of homework completed. The client section of the CCS-BN was used to measure client cognitive and behavioral changes, client engagement in session, and engagement in homework assignments.

Interrater reliability was established using intraclass correlation (ICC), which is conceptualized as the ratio of between-groups variance to total variance (Shrout \& Fleiss, 1979). The ICC was used due to its preference over Pearson's $r$ when sample size is small because Pearson's r overestimates test-retest correlation. A two-way mixed model was used because all raters of interest rated all sessions, resulting in judges being seen as a fixed effect (not as a random sample of all possible raters/judges) and the targets as a random effect, which resulted in the ICC being interpreted as not being generalizable beyond the given judges. The ICC average measure coefficient was interpreted because the mean of all ratings was the unit of analysis. That is, the research design involved averaging multiple ratings for each item. The ICC will approach 1.0 when there is no variance within targets (for a specific session, all raters give the same ratings), indicating total variation in measurements on the Likert scale is due 
Table 2

Reliability (ICC) for Individual Raters During Training and Rating Process

\begin{tabular}{lccccccccc}
\hline & \multicolumn{7}{c}{ Weeks } \\
\cline { 2 - 10 } Rater & 1 & 2 & 3 & 4 & 5 & 6 & 7 & 8 & 9 \\
All & .92 & .96 & .93 & .97 & .97 & .92 & .85 & .91 & .86 \\
R1 & .90 & .95 & .86 & .95 & .93 & .87 & -- & -- & -- \\
R2 & .87 & .94 & .86 & .93 & .91 & .82 & -- & -- & -- \\
R3 & .87 & .90 & .83 & .92 & .91 & .85 & -- & -- & -- \\
R4 & .74 & .91 & .84 & .93 & .90 & .82 & -- & -- & -- \\
\hline
\end{tabular}

solely to the target variable. The ICC for all raters in this study was established to be above 0.90 before the rating of study sessions started. The ICC for individual raters was established to be above .90 before raters started rating sessions and above .82 during the rating process. The ICC for all raters and for individual raters is shown in Table 2.

\section{Procedures}

Definition of sudden gains. A sudden gain (Tang \& DeRubeis, 1999b) was originally defined as a large change in absolute terms (seven points in the Beck Depression Inventory), in relation to symptom severity before the gain (at least $25 \%$ of the pregain's session BDI score), and in relation to symptom fluctuations preceding, and following the gain (the mean BDI score of the three therapy sessions before the gain were significantly higher than the mean BDI score of the three therapy sessions after the gain). The magnitude of seven BDI points as a significant change in depressive symptoms was achieved somewhat arbitrarily. The authors determined this value by examining the frequency distribution plots of between-sessions BDI score changes and noticed a secondary peak, which began at seven BDI points and peaked at nine BDI points, 
concluding that between-session BDI changes greater than seven points may be qualitatively different from smaller ones.

The important aspects of sudden gains definition included absolute magnitude of change, relative magnitude of change, and stability of the change in symptomatology relative to symptom fluctuation. It has been suggested that the second criterion (relative magnitude) be dropped because it has shown minimal impact on sudden gain selection (Tang, DeRubeis, Beberman, \& Pham, 2005). The definition of sudden gain has been traditionally based on between-session changes. Some researchers have utilized datasets that assessed symptom change weekly even when sessions were twice-weekly. The findings of such research indicate that sudden gains are present in equivalent rates when sudden gains are evaluated weekly or twice-weekly (Andrusyna et al., 2006; Gaynor et al., 2003; Hardy et al., 2005; Tang, Luborsky, \& Andrusyna, 2002).

Absolute magnitude. The absolute magnitude aspect has been somewhat debated because of its arbitrariness. A study which examined sudden gains in the treatment of panic disorder used a similar definition of sudden gains, deciding on one standard deviation as a measure of magnitude of symptom change (Pham, 2006). Using one standard deviation has also been questioned in matters of how large a difference must be in order to be considered a significant gain. It is unclear if one, two, or even three standard deviations on a measure would be equivalent to such significant change, although one standard deviation has typically been used as an acceptable measure of magnitude of symptom change.

Stiles et al (2003) were the first to reframe Tang and DeRubeis's (1999b) absolute magnitude criteria using the concepts of clinical significance and statistical reliability. In their study, they examined the presence of sudden gains in the treatment of generalized anxiety disorder and therefore needed to use a measure other than the BDI to measure therapeutic gains. 
Stiles et al noted that a seven-point gain in the BDI was close to the BDI reliable change index (RCI), which has been defined in the literature as a pre-posttreatment difference that, when divided by the standard error of the difference, is equal to 1.96 (for formulas, see Jacobson \& Truax, 1991, p. 14). Stiles et al then used the CORE-SF as a measure of anxiety symptomatology and the RCI formulas to determine the change in absolute magnitude requisite for considering that a sudden gain had occurred.

In 2005, Hardy et al used the Beck Depression Inventory-II to measure sudden gains in cognitive therapy for depression and also used a RCI criterion to determine the absolute magnitude of sudden gains. They found this definition advantageous because it links sudden gain with the widely used concept of reliable change and because it can be applied to a variety of different measures. Subsequent studies examining the presence of sudden gains in the treatment of social phobia and in general clinical practice also derived the absolute magnitude of the gain on the basis of the RCI (Hofmann, Schulz, Meuret, Moscovitch, \& Suvak, 2006; Davies et al., 2006).

Stability. An additional sudden gain criterion, which states that the sudden gain must be a significant change in magnitude in relation to symptom fluctuations preceding and following the gain (the mean BDI score of the three therapy sessions before the gain were significantly higher than the mean BDI score of the three therapy sessions after the gain) (Tang \& DeRubeis, 1999b) has been later reworded as the mean difference between the scores of the three sessions before the gain and the three sessions after the gain expected to be at least 2.78 times greater than the pooled standard deviations of these two groups of sessions' scores (Tang, DeRubeis, Beberman, Pham, 2005). 
It has been expressed that the three pre-gain and three post-gain scores are not independent observations, and that positive autocorrelation effects are likely to inflate the $t$ value, whereas negative autocorrelation effects are likely to deflate the $t$ value, so that the comparison is not a valid inferential test (Vittengl et al., 2005). However, the stability criterion has been shown to be important in defining sudden gains because dropping it in the Stiles et al. (2003) study yielded over three times as many clients with sudden gains and four times as many gains. Because of such importance, all previous sudden gains studies have adopted a similar stability of change criterion.

Hardy (2005) explained that the $t$ test is an arbitrary variance ratio, but due to its importance in sudden gain definition there was a preference of conceptualizing the previous significance criterion as a descriptive statistic, and used the value of $t=2.50$ (slightly relaxed relative to the original sudden gain definition). Tang's (2005) modification of the stability criterion arose from such criticism. The new description of the stability criterion agrees with a more descriptive statistic approach and the authors affirm that the modified stability criterion is mathematically identical to the original stability criterion, but reworded to better follow statistical convention (Tang, De Rubeis, Beberman, \& Pham, 2005).

There has also been a criticism suggesting that even though mathematically the same, the rewording of the stability criterion is statistically different (Kelly, Roberts, \& Ciesla, 2005), which resulted in the use of a modified stability criteria requiring that the gain reflect an improvement of at least 1.5 standard deviations from the individual mean so that it does not simply reflect the individual's normal variation. This modified criterion has been described as more advantageous because the use of the standard deviation accounts for the non-independence of the three preceding and three following sessions, and compensates for the violation of the 
assumption of independence required for the t-test and allows the identification of very early or late sudden gains (Kelly et al., 2007).

The criticism that Tang and DeRubeis' stability criterion makes it impossible to evaluate changes between the first and second sessions, therefore potentially eliminating very early sudden gains, has been addressed by the use of scores of only two sessions before and after the gain if that is all that is available. Sudden gains that took place in sessions one and two have not been considered out of the assumption that such gains differ in nature from later sudden gains because of the unique content of session one (clinical interview or history taking) (Andrusyna et al., 2006; Busch, Kanter, Landes, \& Kohlenberg, 2006; Tang. DeRubeis, Hollon, \& Amsterdan, 2007).

Sudden gain definition in eating disorders. CHEDS scores for all 53 clients and all eligible sessions were evaluated for the possible occurrence of sudden gains. Two mutually exclusive groups of clients were then created, namely those who exhibited sudden gains during the course of treatment and those who did not. In the present study, a sudden gain will be said to have occurred between the critical session $\mathrm{N}$ and $\mathrm{N}+1$ if the gain meets the following criteria:

1. Magnitude: a decrease of 12 points or more in the total CHEDS score, a decrease of 10 points or more in the Eat subscale CHEDS score and a decrease of 12 points or more in the Body subscale CHEDS. The cut off score for the total CHEDS score was determined on the basis of the formulas for the RCI (Jacobson and Truax, 1991) by Hwang and Spangler (in progress). Similar methods were used to create the RCI values for the first criterion of Eat and Body subscales. This critical CHEDS score difference based on the RCI has been chosen based on the standards used in most research studies evaluating the 
existence of sudden gains that did not use the BDI as the measure tracking symptom change.

2. Stability relative to symptom fluctuation: the mean difference between the CHEDS score of the three sessions before the gain and the three sessions after the gain is at least 2.78 times greater than the pooled standard deviations of these two groups of sessions' CHEDS scores. The second criterion for Eat and Body subscale was the same as the total CHEDS sudden gain second criterion just described. This criterion was chosen to account for the stability of the sudden gain in the same manner that has been done by most sudden gains investigators. When Tang and DeRubeis (2005) modified the original third criterion, in place of the t-test with a critical value of 2.78 , they framed 2.78 as a multiplier to require that the mean difference between the pre-gain and post-gain scores exceed 2.78 times the pooled standard deviation of the two groups in a manner that does not violate the assumption of independence required for t-tests and allows the inclusion of sudden gains that occur very early or very late in treatment (i.e., within three sessions of initiating of terminating treatment). This new definition has been more frequently used and more accepted than any other. As in previous studies, when applying the stability criterion to session 2, session $\mathrm{N}-2$ will not be used; furthermore, when applying this criterion to the third to the last session, session $\mathrm{N}+3$ will not be used.

Session ratings. Therapy sessions were categorized into groups based on the sudden gain analysis including pre-pregain sessions, pre-gain sessions, and post-sudden gain sessions. Pre-gain sessions are considered to be critical sessions and were therefore rated; the pre-pregain sessions were used as control sessions because they are close in time to the pre-gain sessions and 
because they occur before the sudden gain. Post-gain sessions were rated to evaluate the phenomena that followed sudden gains.

Each critical and control sessions were rated independently by two raters and the average of the values obtained by the two raters was used as the final score. The raters were four clinical psychology graduate students. Their experience in cognitive behavioral therapy ranged from one to four years. Training in coding CBT sessions consisted of reviewing the measure with its primary author, and practicing rating random CBT sessions from the dataset until an interrater reliability of .90 was achieved. Once raters started to rate sessions identified for the current study, they all rated one selected session a week to verify that interrater reliability was being maintained during the rating process. The ratings were completed while the raters listened to and watched the videotapes of the selected therapy sessions. Raters were blind to type of session they were rating (i.e., pre-pregain, pre-gain or post-gain), to client symptom change, and client treatment outcome. To minimize the impact of interrater differences, the pre-gain session and the pre-pregain session of a given therapist-client dyad were assigned to the same pair of raters.

\section{Results}

\section{Description of Sudden Gains}

Out of the 745 between-sessions intervals of the 53 clients, 41 sudden gains experienced by 28 different clients were identified based on total CHEDS score, resulting in 53\% of clients experiencing at least one sudden gain. Although total CHEDS sudden gains were observed throughout therapy, the median $50 \%$ fell between the $3^{\text {rd }}$ and the $12^{\text {th }}$ sessions, with session 8 being the median and session 2 the mode pregain session. The percentage of sudden gains occurring between sessions 4 and 10 was $28 \%$. Sub analysis were conducted to explore whether or not the prevalence of total CHEDS sudden gains was different when sudden gains were 
defined as weekly gains in comparison to between-session gains (since therapy sessions are offered twice a week for the first three weeks of treatment). Out of the 619 weekly session intervals of the 53 clients, 33 weekly sudden gains were identified, experienced by 21 different clients, resulting in $40 \%$ of clients experiencing at least one sudden gain. Weekly sudden gains were also observed throughout therapy, where the median $50 \%$ fell between the $3^{\text {rd }}$ and the $9^{\text {th }}$ week of treatment, with week 6 being the median and week 3 the mode pregain session. The average total CHEDS sudden gain is shown in Figure 2. All sudden gains' session N (pregain session) total CHEDS scores were averaged and this process was repeated for sessions $\mathrm{N}-2, \mathrm{~N}-1$, $\mathrm{N}+1, \mathrm{~N}+2$, and $\mathrm{N}+3$. To put these means in context, the average first- and last-session total CHEDS scores of all the clients who experienced sudden gains is also displayed in Figure 2.

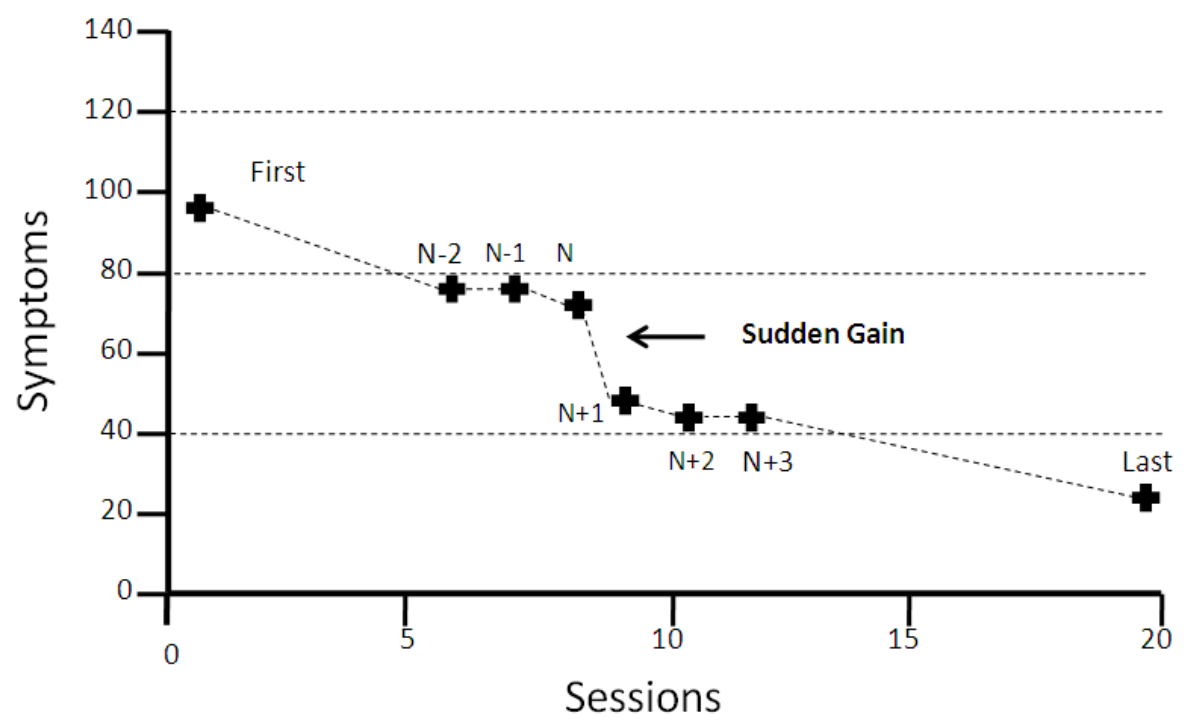

Figure 2. The average total CHEDS sudden gain. CHEDS scores shown for sessions $\mathrm{N}-2, \mathrm{~N}-$ $1, \mathrm{~N}, \mathrm{~N}+1, \mathrm{~N}+2, \mathrm{~N}+3$ are the means of the corresponding sessions from the 41 sudden gains in the sample, with session $\mathrm{N}$ as the pre-gain session. The figure also shows the means of CHEDS scores from the first and last sessions for all of the clients who experienced sudden gains. 
Subscale sudden gains. Out of the 745 between-session intervals of the 53 clients, 45 subscale sudden gains experienced by 33 different clients were identified, resulting in $62 \%$ of clients experiencing at least one subscale sudden gain. Out of the 745 between-session intervals of the 53 clients 24 Eat subscale sudden gains experienced by 20 different clients were identified, resulting in $38 \%$ of clients experiencing at least one Eat subscale sudden gain. Although Eat subscale sudden gains were observed throughout therapy, the median $50 \%$ fell between the $3^{\text {rd }}$ and the $11^{\text {th }}$ sessions, with session 5 being the median and session 2 the mode pre-gain session. Out of the 745 between-sessions intervals of the 53 clients, 21 Body subscale sudden gains experienced by 16 different clients were identified, resulting in $30 \%$ of clients experiencing at least one Body subscale sudden gain. Body subscale sudden gains were also observed throughout therapy, but the median $50 \%$ fell between the $5^{\text {th }}$ and the $15^{\text {th }}$ sessions, with session 10 being the median and session 15 the mode pre-gain session. The average subscale sudden gain is shown in Figure 3. All subscale sudden gains' session N (pre-gain session) total CHEDS scores were averaged and this process was repeated for sessions $\mathrm{N}-2, \mathrm{~N}-1, \mathrm{~N}+1, \mathrm{~N}+2$, and $\mathrm{N}+3$. To put these means in context, the average first- and last-session CHEDS subscale scores of all the clients who experienced subscale sudden gains is also displayed in Figure 3.
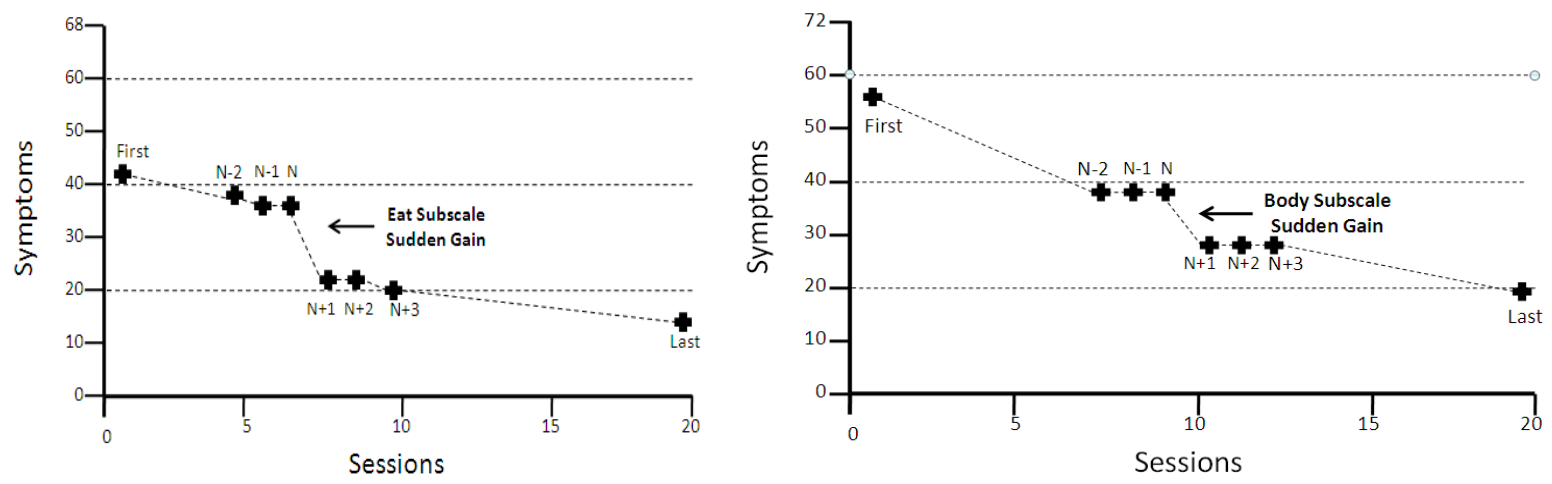

Figure 3. The average subscale sudden gain. Eat subscale sudden gain on the left and Body subscale sudden gain on the right. 


\section{Impact of Sudden Gains at the End of Treatment}

The mean magnitude of all identified total CHEDS sudden gains was 23.9 CHEDS points $(S D=15.29)$. The mean of the total CHEDS improvement accomplished by the entire CBT treatment was 37.2 points $(S D=31.5)$ for all of the clients and 57.2 points $(S D=20.9)$ for the clients who experienced sudden gains. Thus, for clients who experienced total CHEDS sudden gains, each sudden gain accounted for an average of $42 \%$ of their total symptom reduction. Given that several clients experienced more than one total CHEDS sudden gain, sudden gains experienced per client accounted on average for $69 \%$ of their total symptom reduction. To confirm that the sudden gains represented actual stable change and not transient variability in symptoms, presence of symptom reversals were investigated with reversals being defined as a loss of $50 \%$ or more of the symptom improvement resulting from the sudden gain. For example, if a client's pre-gain session CHEDS score was 80 and after-gain session CHEDS score was 60, whenever his or her CHEDS score returned to 70 or higher a reversal was considered to have occurred. Out of the 28 clients who experienced sudden gains, 13 (28\%) clients experienced a reversal before the end of therapy. Out of 42 sudden gains, $21(50 \%)$ sudden gains were reversed.

At post-treatment, all clients who had experienced sudden gains (having experienced symptom reversal or not) achieved better outcomes that those who did not experience sudden gains. The mean post-treatment CHEDS score for those who experienced total CHEDS sudden gains was $33(S D=19.9)$, which falls well below the clinical CHEDS cutoff score of 60 . The sudden gains clients' outcomes were also significantly better than the outcomes of clients who did not experience sudden gains (see Figure 4). At the beginning of therapy, the two groups of clients evidenced essentially equal levels of eating disorder symptomatology: The mean total 
CHEDS score was 86.5 for clients who later experienced total CHEDS sudden gains $(n=29, S D$ $=22.3)$ and 87.7 for those who did not $(n=23, S D=25.2)$. This pre-treatment difference was not a significant difference, $t(51)=-0.18, p=.86$. At post-treatment, the mean CHEDS score of the clients who did not experience a total CHEDS sudden gain was 69.8 points $(S D=31.2)$, which was significantly higher than the mean $(M=32.4, S D=21.9)$ for the clients who experienced a sudden gain, $t(51)=-5.11, p=.02$. The effect size of the difference was substantial $(d=-1.43)$. It should also be noted that the magnitude of the difference in posttreatment scores $(36.8)$ was close to two-thirds $(65 \%)$ of the average magnitude of the sudden gains (23.9).

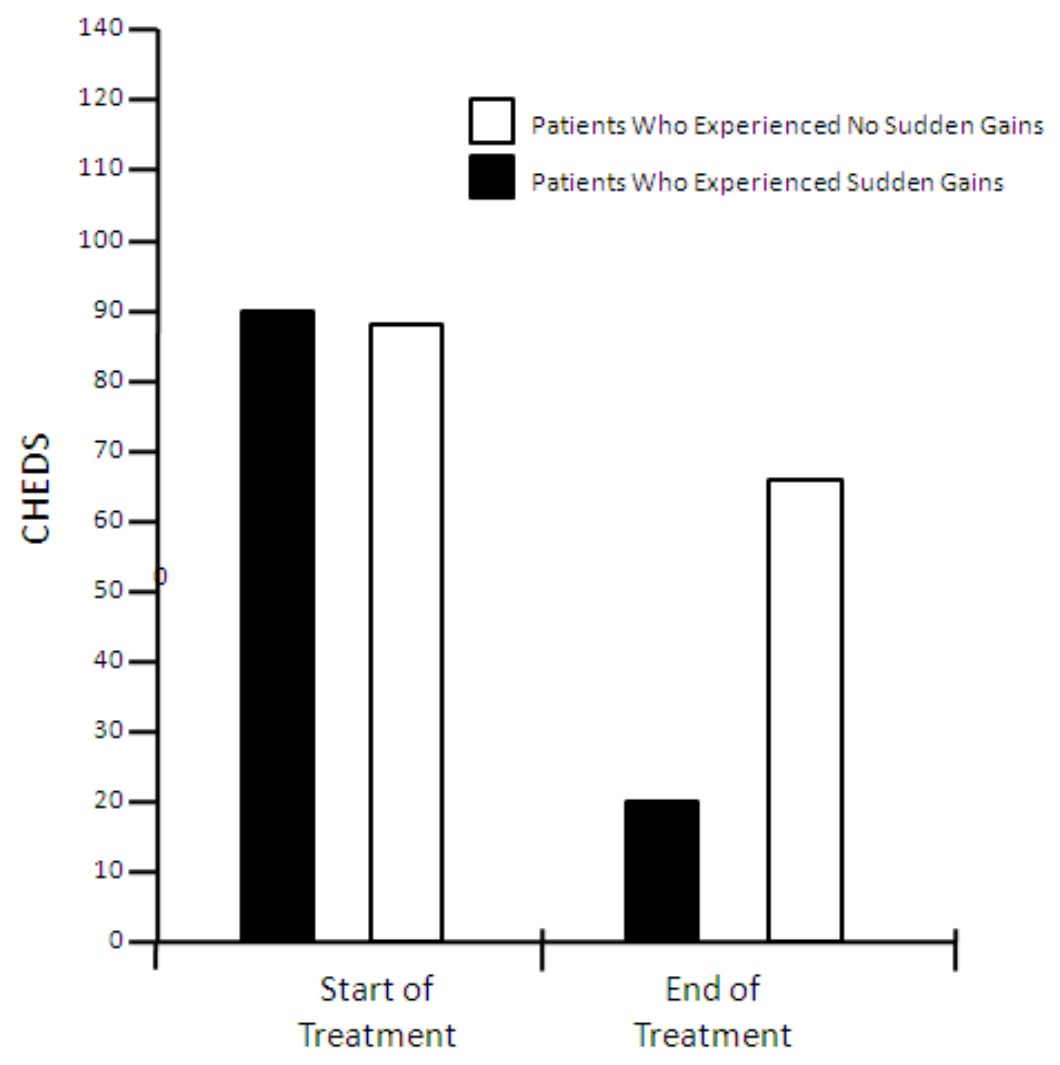

Figure 4. Outcome comparison of clients who experienced Total CHEDS sudden gains those who did not experience sudden gains. 
Eat subscale sudden gains. The mean magnitude of all identified Eat subscale sudden gains was 14.9 CHEDS points $(S D=6.4)$. The mean of the Eat subscale CHEDS improvement was $18.3(S D=14.28)$ points for all of the clients and $26.5(S D=11.9)$ points for the clients who experienced Eat subscale sudden gains. Thus, for clients who experienced Eat subscale sudden gains, the sudden gains accounted for an average of $56 \%$ of their total eating related symptom reduction and for an average of $27.2 \%$ of their total eating disorder symptom reduction. To confirm that Eat subscale sudden gains reflected actual stable change, and not transient symptom fluctuation, the occurrence of reversals occurring before the end of therapy was assessed. Out of the 20 clients who experienced sudden gains, six experienced a reversal before the end of therapy. Out of 24 Eat subscale sudden grains, six sudden gains were reversed.

At post-treatment, all clients who had experienced Eat subscale sudden gains (having experienced symptom reversal or not) experienced good outcomes. Their mean post-treatment total CHEDS score was $40.3(S D=26.8)$. These clients' outcomes, however, were not significantly better than the outcomes of clients who did not experience Eat subscale sudden gains (see Figure 5). At the beginning of therapy, the two groups of clients evidenced similar levels of eating disorder symptomatology. The mean pretreatment CHEDS Eat subscale score was 41.6 for clients who later experienced Eat subscale sudden gains $(n=20, S D=12.7)$ and 34.2 for those who did not $(n=33, S D=14)$. This difference was not a significant difference, $t(51)=1.92, p=.95$. At posttreatment, the mean CHEDS Eat subscale score of clients who did not experience a sudden gain was 20.9 points $(S D=15.5)$, which was not significantly different from the mean $(M=15.2, S D=14.9)$ for the clients who experienced a sudden gain, $t(51)=-.67$, $p=.92$. It should also be noted that the magnitude of the difference in post-treatment scores (12.9) represents over three-fourths (86.6\%) of the average magnitude of the sudden gains (14.9). 
Body subscale sudden gains. The mean magnitude of all identified Body subscale sudden gains was 19.3 CHEDS points $(S D=12.1)$. Thus, for clients who experienced Body subscale sudden gains, the sudden gains accounted for an average of $58.2 \%$ of their total body related symptom reduction and for an average of $32.1 \%$ of their total eating disorder symptom reduction. To confirm that Body subscale sudden gains reflected stable change, and not transient symptom fluctuation, occurrence of symptom reversals was assessed. Out of the 16 clients who experienced Body subscale sudden gains, 10 clients experienced a reversal before the end of therapy. Out of 21 Body subscale sudden grains, 10 sudden gains were reversed.

At post-treatment, all clients who had experienced Body subscale sudden gains (having experienced symptom reversal or not) achieved good outcomes. Their mean posttreatment total CHEDS score was 32.6 ( $S D=21.8$ ). These clients' outcomes were also significantly better than the outcomes of clients who did not experience Body subscale sudden gains (see Figure 5). At the beginning of therapy, the two groups of clients evidenced similar levels of eating disorder symptomatology. The mean pretreatment CHEDS Body subscale score was 55.1 ( $S D=10.2)$ for the 16 clients who later experienced Body subscale sudden gains and $48.1(S D=12.8)$ for the 37 clients who did not experience sudden gains. This difference was not a significant difference, $t(51)=1.93, p=.53$. At post-treatment, the mean CHEDS Body subscale score of the clients who did not experience a sudden gain was 34.2 points $(S D=19.9)$, which was significantly higher than the mean $(M=21.7, S D=12.5)$ for the clients who experienced a sudden gain, $t(51)$ $=-2.30, p=.01$. The effect size of the difference was medium $(d=-0.64)$. The magnitude of the difference in post-treatment scores (27.0) represents about two-thirds (64\%) of the average magnitude of the Body subscale sudden gains (19.3). 


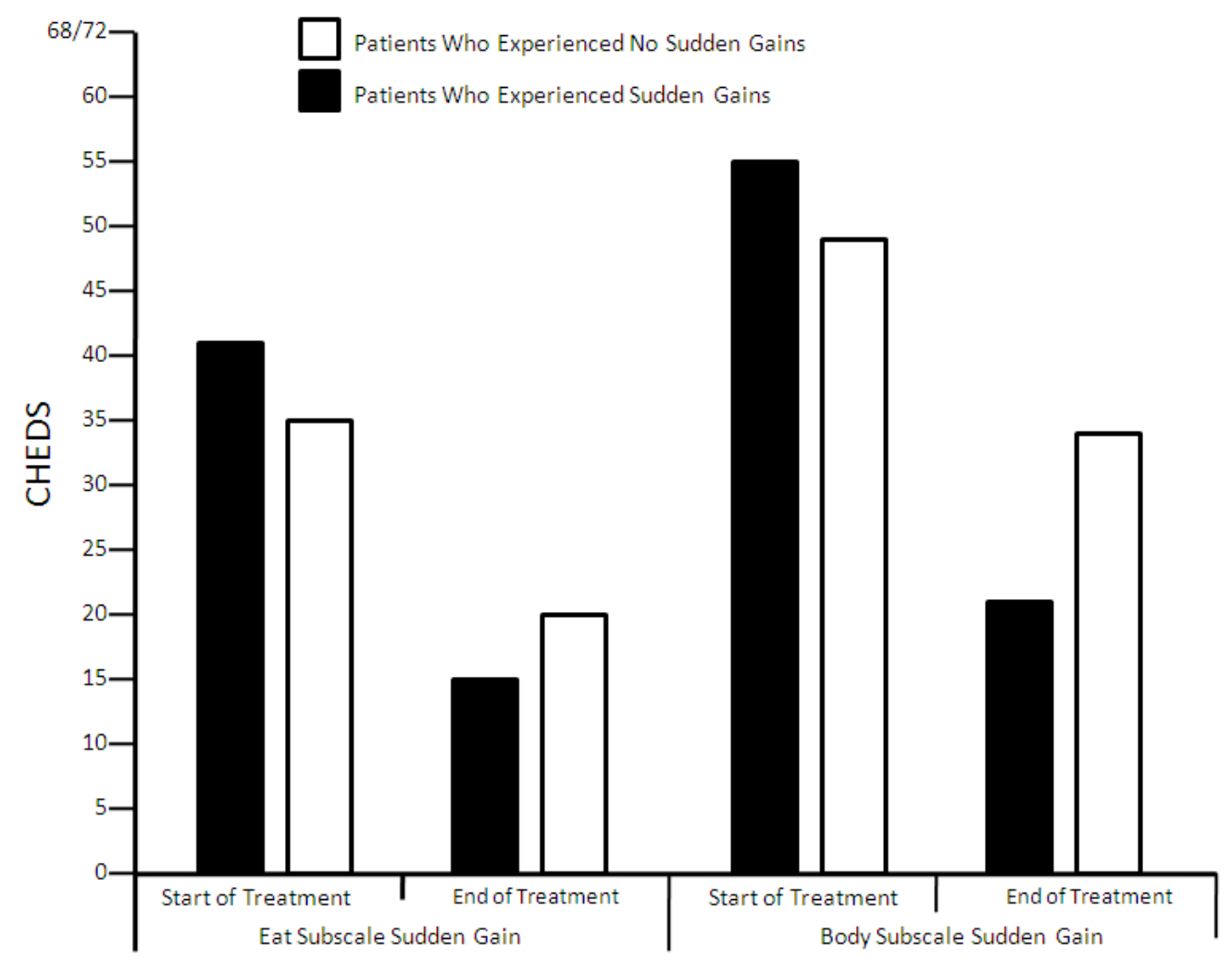

Figure 5. Outcome comparison of clients who experienced Eat subscale and Body subscale sudden gains compared with those who did not experience sudden gains.

\section{Differentiation of Critical Sudden Gain Sessions}

CHEDS total score sudden gains. To examine how the critical sessions identified for total CHEDS sudden gains may be different from other therapy sessions in the current study, a multivariate omnibus test $(\alpha=0.05)$ was conducted. The multivariate omnibus test was significant (Wilks' Lambda $F=2.20, p=0.05$ ), indicating that the level of purported predictors of sudden gains (i.e. therapist interventions, client mechanisms) in critical sessions was different between the pre-pregain session and the pregain session. Follow up univariate tests indicated a significant difference in levels of therapist cognitive interventions $(F=4.0, p=0.05)$, therapist empathy $(F=6.96, p=0.01)$, client cognitive change $(F=6.70, p=0.01)$, and client motivation $(F=8.63, p=0.006)$. All of these were reflective of greater levels of these variables in the pre- 
gain session compared to the pre-pregain session. Effect sizes were small (Cohen's $d=-0.34$; Cohen, 1977).

Regression analyses were also conducted to evaluate if changes in purported predictors of sudden gains actually predicted the amount of change that occurred during the total CHEDS sudden gain. The change in each purported predictor between the pre-pregain session (N-1) and the pre-gain session $(\mathrm{N})$ was used to investigate whether change in identified predictors between those two sessions predicted the amount of the sudden gain. A significant model emerged $\left(F(11,8)=6.48, p<0.000\right.$. Adjusted $\left.R^{2}=.65\right)$. Specifically, decreases in client homework significantly predicted the amount of change that occurred in total CHEDS sudden gains, $b=$ $.22, t(2)=-2.024, p=.002$.

Eat subscale sudden gains. To examine if the critical sessions of Eat subscale sudden gains were different from other therapy sessions in the current study, a multivariate omnibus test was conducted. The multivariate omnibus test was not significant (Wilks' Lambda $F=1.17, p=$ 0.40), indicating that the level of purported predictors of sudden gains (i.e., therapist interventions, client mechanisms) in Eat subscale critical sessions were not different between the pre-pregain session and the pregain session. Regression analyses were also conducted to evaluate if changes in purported predictors predicted the amount of change in the Eat subscale sudden gain. The difference between the pre-pregain session $(\mathrm{N}-1)$ and the pre-gain session $(\mathrm{N})$ of each purported sudden gain predictor identified in hypothesis 2 were used to investigate whether changes in identified predictors between those two sessions predicted the amount of sudden gain in the Eat subscale. A marginally significant model emerged $(F(10,9)=2.799, p=$ 0.07. Adjusted $R^{2}=.486$ ). Significant predictors included increases in therapist cognitive 
interventions $(b=.573, t(10)=2.473, p=0.035)$ and decreases in levels of therapist empathy $(b$ $=-.835, t(10)=-2.627, p=0.028)$.

Body subscale sudden gains. To examine the how the critical sessions for the Body subscale may be different from other therapy sessions a multivariate omnibus test was conducted for the Body subscale of the CHEDS. The multivariate omnibus test was not significant (Wilks' Lambda $\mathrm{F}=1.020, p=0.48)$, indicating that the level of purported predictors of sudden gains (i.e., therapist interventions, client mechanisms) in Body subscale critical sessions were not different between the pre-pregain session and the pregain session. Regression analyses were also conducted to evaluate if changes in purported predictors predicted the amount of change occurring during Body subscale sudden gains. The difference between the pre-pregain session $(\mathrm{N}-1)$ and the pre-gain session $(\mathrm{N})$ of each purported predictor identified in hypothesis 2 was used to investigate whether changes in predictors between those two sessions predicted the amount of Body subscale sudden gain. A significant model emerged $(\mathrm{F}(11,8)=4.621, p=0.019$. Adjusted $\left.\mathrm{R}^{2}=.677\right)$. More specifically, increases in changes in client cognition $(b=.803, t(11)$ $=3.598, p=.007)$ and decreases in client homework $(b=-.725, t(11)=-3.597, p=.007)$ both significantly predicted the amount of change that occurred in Body subscale sudden gains.

\section{Phenomena after Sudden Gain Sessions}

CHEDS total sudden gains. To examine the phenomena after total CHEDS sudden gains, two multivariate omnibus tests were conducted. The first test was designed to compare level of purported predictors of sudden gains (i.e., therapist interventions, client mechanisms) between the pre-pregain and the post-gain sessions. The first multivariate omnibus test was significant (Wilks' Lambda $F=5.238, p=0.00$ ), indicating that the level of purported predictors of sudden gains (i.e., therapist interventions, client mechanisms) was significantly different 
between the pre-pregain session and the post-gain session. Follow up univariate tests indicated significantly different levels of therapist empathy, client behavioral change, client cognitive change, and client motivation, with values for these variables increasing after the sudden gain. Effect sizes ranged from medium to large (Cohen's $d=-0.55$ to $d=-0.94$; Cohen, 1977). Significant variables are shown in Table 3.

The second multivariate omnibus test was designed to compare level of purported predictors of sudden gains (i.e., therapist interventions, client mechanisms) between the pre-gain and the post-gain sessions. The test was marginally significant (Wilks' Lambda $F=1.920, p=$ 0.092), indicating that the level of purported predictors of sudden gains (i.e., therapist Table 3

Effect Sizes for Significant Variables after Total CHEDS Sudden Gains

\begin{tabular}{lccc}
\hline \multicolumn{4}{c}{ Pre-PreGain and post-Gain Sessions Comparison } \\
\hline Predictor Variable & $F$ & $d$ & Cohen's $d$ \\
\hline Therapist Empathy & 9.011 & .005 & -.55 \\
Client Behavioral Change & 11.838 & .002 & -.58 \\
Client Cognitive Change & 31.522 & .000 & -.94 \\
Client Motivation & 23.846 & .000 & -.71 \\
\hline & Pre-Gain and Post-Gain Sessions Comparison & \\
\hline Predictor Variable & $F$ & $d$ & Cohen's $d$ \\
\hline Client Behavioral Change & 8.261 & .007 & -.50 \\
Client Cognitive Change & 21.149 & .000 & -.58 \\
Client Motivation & 8.675 & .006 & .079 \\
Client Homework & 3.278 & & -.37 \\
\hline
\end{tabular}


interventions, client mechanisms) in critical sessions was marginally different between the pregain session and the post-gain session. Follow up univariate tests indicated significant differences in levels of therapist empathy, client behavioral change, client cognitive change, and client motivation, with the value for these variables increasing after the sudden gain. Effect sizes ranged from low to medium (Cohen's $d=-0.21$ to $d=-0.58$; Cohen, 1977). Significant variables and effect sizes are shown in Table 3.

Eat subscale sudden gains. To examine the phenomena after Eat subscale sudden gains, two multivariate omnibus tests were conducted. The first test compared level of purported predictors of sudden gains (i.e., therapist interventions, client mechanisms) between the prepregain and the post-gain sessions. The test was marginally significant (Wilks' Lambda $F=2.56$, $p=0.07$ ), indicating that the level of purported predictors of sudden gains (i.e., therapist interventions, client mechanisms) in critical sessions was marginally different between control sessions and the post-gain session. Follow up univariate tests indicated differences in levels of therapist cognitive interventions $(F=5.82, p=.02$, Cohen's $d=-0.46)$, therapist empathy $(F=$ $10.24, p=.005$, Cohen's $d=-0.82)$, client behavioral change $(F=14.59, p=.001$, Cohen's $d=-$ 0.77), client cognitive change $(F=25.83, p=.00$, Cohen's $d=-0.94)$, and client motivation $(F=$ $11.24, p=.003$, Cohen's $d=0.73$ ), with the value for these variables increasing after the sudden gain. The second test compared the level of purported predictors of sudden gains between the pre-gain and the post-gain sessions. This test was not significant (Wilks' Lambda $F=1.23, p=$ 0.37), indicating that the level of purported predictors of sudden gains (i.e., therapist interventions, client mechanisms) in the post-gain session was not different from the critical session. 
Body subscale sudden gain. To examine the phenomena after Body subscale sudden gains, two multivariate omnibus tests were conducted. The first test compared the level of purported predictors of sudden gains (i.e., therapist interventions, client mechanisms) between the pre-pregain and the post-gain sessions. The test was significant (Wilks' Lambda $F=3.27$, $p=0.04$ ), indicating that the level of purported predictors of sudden gains (i.e., therapist interventions, client mechanisms) in critical sessions was significantly different between the prepregain session and the post-gain session. Follow up univariate tests indicated differences in levels of therapist empathy, client behavioral change, client cognitive change, and client motivation, with the value for these variables increasing after the sudden gain. Effect sizes

Table 4

Effect Sizes for Significant Variables after Body Subscale Sudden Gains

\begin{tabular}{lccc}
\hline \multicolumn{4}{c}{ Pre-PreGain and post-Gain Sessions Comparison } \\
\hline Predictor Variable & $F$ & $d$ & Cohen's $d$ \\
\hline Therapist Empathy & 8.245 & .010 & -.69 \\
Client Behavioral Change & 4.492 & .047 & -.53 \\
Client Cognitive Change & 17.873 & .000 & -.93 \\
Client Motivation & 11.060 & .004 & -.85 \\
\hline & Pre-Gain and Post-Gain Sessions Comparison & \\
\hline Predictor Variable & $F$ & $d$ & Cohen's $d$ \\
\hline Client Behavioral Change & 7.228 & .015 & -.65 \\
Client Cognitive Change & 23.612 & .000 & -.97 \\
Client Motivation & 8.223 & .010 & .005 \\
Client Homework & 9.812 & -.57 \\
\hline
\end{tabular}


ranged from medium to high (Cohen's $d=-0.53$ to $d=-0.93$; Cohen, 1977). Significant variables and effect sizes are shown in Table 4.

The second test compared the level of purported predictors of sudden gains (i.e., therapist interventions, client mechanisms) between the pre-gain and the post-gain sessions. The test was marginally significant (Wilks' Lambda $F=2.67, p=0.06$ ), indicating that the level of purported predictors of sudden gains in the post-gain session was marginally different between the pre-gain session and the post-gain session. Follow up univariate tests indicated differences in levels of client behavioral change, client cognitive change, client motivation, and client homework, with the value for these variables increasing after the sudden gain. Effect sizes ranged from medium to high (Cohen's $d=-0.50$ to $d=-0.97$; Cohen, 1977). Significant variables and effect sizes are shown in Table 4.

\section{Discussion}

The current study focused on three types of sudden gains, total CHEDS sudden gains which encompass all eating disorder symptomatology, and two subscale sudden gains (Eat and Body) which encompass specific separate domains of eating disorder symptomatology. The existence of distinct and independent Eat and Body subscale sudden gains support the uniqueness of these symptom dimensions and are consistent with cognitive behavioral theory and treatment of eating disorders.

\section{Total CHEDS Sudden Gain}

Similar to sudden gain findings previously reported, over $50 \%$ of clients experienced at least one sudden gain and demonstrated significantly better acute outcomes, partially supporting hypothesis one. Each total CHEDS sudden gain accounted for $42 \%$ of clients' total symptom improvements, with $46 \%$ of sudden gainers experiencing sudden gain reversal before the end of 
therapy, and with $28 \%$ of sudden gains taking place between the $4^{\text {th }}$ and $10^{\text {th }}$ sessions. These findings stand in contrast to the original sudden gains findings in the treatment of depression previously reported by Tang and DeRubeis (1999) where no more than $17 \%$ of sudden gainers experienced sudden gain reversal before the end of therapy, and with $50 \%$ of sudden gains taking place between the $4^{\text {th }}$ and $10^{\text {th }}$ sessions. Despite the discrepancies between the current findings and those of Tang and DeRubeis, sudden gains in the treatment of eating disorders did represent a stable symptom improvement. Clients who experienced a sudden gain, regardless of whether or not a reversal occurred, demonstrated significantly better post-treatment outcomes than those of clients who did not experience a sudden gain. This finding suggests that sudden gains are not transient and inconsequential fluctuations. Instead, they are indicative of substantial, longlasting symptom improvement. Because the benefits experienced by clients with sudden gains appeared to be maintained through the end of treatment, understanding sudden gains might be important for understanding at least how the majority of responders achieved recovery.

The pre-gain session, or the critical session, consisted of higher levels of therapist cognitive interventions, therapist empathy, client cognitive change, and client motivation when compared to control therapy sessions. Therefore, hypothesis two was also partially supported, indicating that among the variables investigated in the pregain sessions, therapist cognitive interventions, relational interventions, client cognitive changes, and client motivation increases were the most likely triggers of the sudden gains. These findings support the idea that both therapist and client do some things differently in the session preceding sudden gains. Specifically, therapists have increased levels of cognitive interventions and empathy, and clients experience more cognitive changes and increased motivation. In addition, regression analyses indicated that decreases in client homework predicted the amount of change that occurred during 
the total CHEDS sudden gain. This is an interesting finding because it suggests that clients' decrease in homework may be one factor that prompts therapist and client to have a different experience in the therapy session occurring just prior to the sudden gain.

\section{Subscale Sudden Gains}

Two different kinds of sudden gains in CBT for eating disorders were found to exist, one taking place due to changes in eating related thoughts, feeling, and behaviors (e.g. feeling like one cannot stop eating, eating more rapidly than normal, restricting the amount of food eaten, vomiting, etc) and another due to changes in body related thoughts, feelings, and behaviors (e.g. body checking, body dissatisfaction, desire to become thinner, etc.), fully supporting hypothesis three. About $43 \%$ of clients experienced at least one subscale sudden gain, with $18 \%$ of those experiencing both Eat and Body subscale sudden gains. The existence of two different types of sudden gains in the cognitive behavioral treatment of eating disorders is significant because it supports that eating disorders are composed of two different set of symptoms, one related to eating and another related to body.

Eat subscale sudden gains. Eat subscale sudden gains were experienced by $38 \%$ of clients, with $50 \%$ of Eat subscale sudden gains falling between the $3^{\text {rd }}$ and the $11^{\text {th }}$ sessions, with session two being the mode pregain session and session five being the median pregain session. Even with $25 \%$ of Eat subscale sudden gains being reversed, at post-treatment, all clients who had experienced Eat subscale sudden gains (whether a symptom reversal occurred or not) experienced good outcomes. Outcome differences for clients who experienced Eat subscale sudden gains and those who did not were not significant. At the same time, the magnitude of the difference in post-treatment scores between the two groups represented over three-fourths of the average magnitude of Eat subscale sudden gains. The pregain session, or the critical session, of 
Eat subscale sudden gains was marginally different from control sessions. Hypothesis four was not supported because Eat sudden gains were not found to be primarily predicted by client behavioral changes (e.g. engagement in regular eating). Instead, regression analyses identified increases in therapist cognitive interventions and decreases in levels of therapist empathy as predictors of the amount of change that occurred in Eat subscale sudden gains.

Body subscale sudden gains. Body subscale sudden gains were experienced by $30 \%$ of clients with $50 \%$ of Body subscale sudden gains falling between the $5^{\text {th }}$ and the $15^{\text {th }}$ sessions, with session 15 being the mode pregain session and session 10 being the median. However, $47 \%$ of Body subscale sudden gains were reversed. At post-treatment, all clients who had experienced Body subscale sudden gains (whether a symptom reversal occurred or not) achieved good outcomes. Body subscale sudden gainers' outcomes were significantly better than the outcomes of clients who did not experience Body subscale sudden gains. Hypothesis five was supported, with cognitive changes (i.e. changes in maladaptive beliefs about appearance) being the primary identified predictor of Body subscale sudden gains. Regression analyses identified increases in client cognitive change and decreases in client homework as significant predictors of the amount of change that occurred in Body subscale sudden gains.

\section{Sudden Gains and the Cognitive Model}

The findings of the present study are consistent with the cognitive model, which emphasizes the importance of cognitive change in producing clinical improvement. Cognitive theory proposes that individuals' affect and behavior are largely determined by how they think about the world and that correction of faulty thinking leads to clinical improvements (Beck, 1979). According to the cognitive behavioral theory of eating disorders, an overvaluation of body shape and weight leads to many eating disorder symptoms (i.e. frequent weighing or 
avoidance of weighing, repeated checking or scrutinizing of the body, and feeling fat). In fact, eating disorders have been described as primarily disorders of cognition (Fairburn, 2008) due to the distinct core cognitive psychopathology (i.e., the overvaluation of shape and weight). The cognitive behavioral treatment used in this study follows the cognitive model and focuses on eating disorders as stemming from dysfunctional beliefs about the body and about food that lead to dissatisfaction with shape and weight, which then leads to the development of rigid eating patterns aimed at controlling and changing shape and weight. Thus, the findings that therapist cognitive interventions and client cognitive changes were among the most significant predictors of both total CHEDS sudden gains and of Body subscale sudden gains particularly support the cognitive model. The finding that Eat sudden gains were primarily predicted by increases in therapist cognitive interventions furthers support the importance of cognitive interventions in changing many eating related symptoms (e.g. dietary restriction, binge eating, and compensatory behaviors). In addition, increases of client cognitive change as a predictor of Body subscale sudden gains demonstrate the importance of cognitive changes in the complete recovery of eating disorders.

Because Body subscale sudden gains had a significant impact at the end of treatment when compared to Eat subscale sudden gains, it is possible that Body sudden gains are more central to eating disorder recovery due to their relationship to core cognitive eating disorder psychopathology. The previous literature has indicated early change in purging as the most consistently identified predictor of outcome for eating disorders (Fairburn, Agras, Walsh, Wilson, \& Stice, 2004), which contrasts with the findings in this study that suggest that gains in body-related variables are more potent predictors of outcome than eating-related changes. Many clients with eating disorders do not engage in purging, which makes it difficult to use change in 
purging as a reliable predictor of outcome. Therefore, body-related symptoms seem to be more ubiquitous and more central to outcome among those with eating disorders than is purging, and changes in body-related symptoms may be a better outcome predictor for clients receiving cognitive-behavioral therapy for eating disorders.

\section{Sudden Gains and the Stages of Eating Disorder Treatment}

The findings of the present study are consistent also with the structure and format of the cognitive behavioral treatment protocol for eating disorders. The finding that Eat subscale sudden gains happen earlier in therapy when compared to Body subscale sudden gains also reflects the structure and format of the cognitive behavioral treatment of eating disorders and supports treatment theory about how eating disorder recovery progresses. The treatment protocol used in this study, as developed by Fairburn (2008), is divided into four stages. Each stage focuses on specific eating disorder symptomatology. Stage One is aimed at eating psychopathology, with the intention to reduce and eventually eliminate excessive dietary restriction, binge eating, and compensatory behaviors. Stage One starts in session one and ends in session six, indicating that both the mode and the median Eat subscale sudden gain falls within stage one. Stage Three of treatment focuses on body psychopathology, with the intention to modify client dysfunctional beliefs about body shape and weight that contribute to body dissatisfaction and dietary restriction, having a great emphasis on changing body image. Stage Three starts in session nine and ends in session 17, encompassing both the mode and the median Body subscale sudden gain. Simply put, changes in eating- and body- related symptoms do not change in unison; rather, changes in eating-related symptoms are most likely to occur during stage one of treatment and changes in body-related symptoms are most likely to occur during stage three of treatment as predicted by the treatment model. 
Additionally, the decrease in homework just prior to the critical session which predicted both total CHEDS and Body subscale sudden gains, although initially counterintuitive may also support the treatment protocol used in this study. When the time courses of client's homework, client cognitive changes and severity of eating disorder symptomatology are synchronized, as shown in Figure 6, it demonstrates that a decrease in the amount of homework completed by the client preceded an increase in the amount of cognitive changes, which in turn preceded a decrease in eating disorder symptomatology. This finding is supported by the treatment protocol used in this study, where the non-completion of homework is addressed with perplexity and followed by the exploration of the client's reasons for not completing homework (Fairburn, 2008). If the client had continued to do homework as usual, most likely the pre-gain session would have been more similar to other therapy sessions. However, when the client did not complete homework, the focus of the session became different from other sessions. It is possible that clients who experience a gradual recovery, instead of a sudden gain recovery, complete homework steadily throughout therapy. Homework completion is part of clients' motivation, with the supposition that more motivated clients complete more homework. With regard to total CHEDS sudden gains, client motivation increases in the critical session, which once more supports the idea that because homework decreased, client motivation, homework completion and related distorted thinking were addressed in the critical session, which in turn led to an increase in cognitive changes, greater motivation, and subsequent homework completion. It is important to notice that the in-session experience prior to the sudden gain (during the critical session) bring significant changes that allowed clients to become more motivated, to fully participate in treatment, to be more successful in engaging in out-of-session therapeutic activities, and to make greater progress. The critical session and the sudden gain may be crucial 


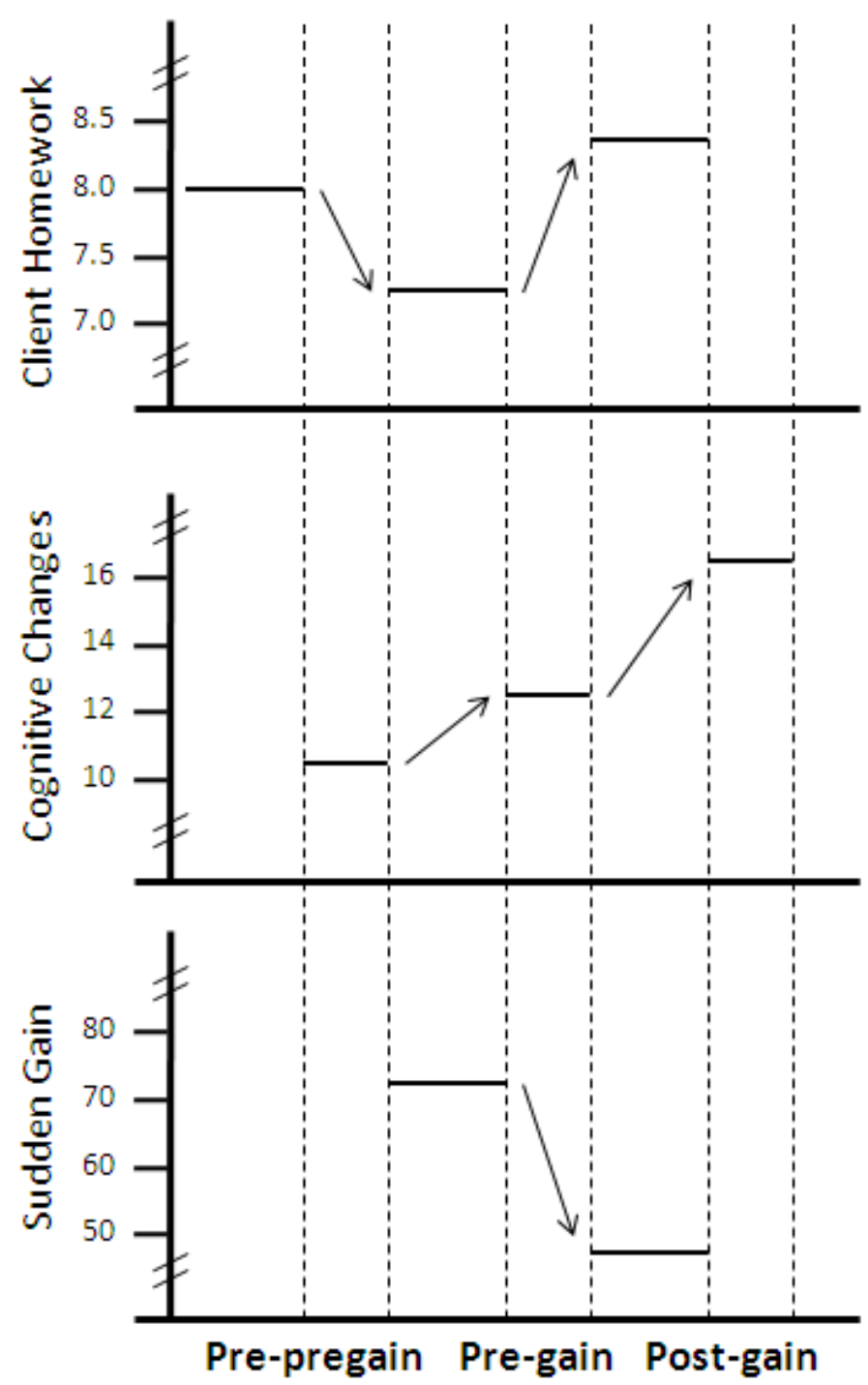

Figure 6. The synchronized time courses of client's homework, client cognitive changes and severity of eating disorder symptomatology demonstrates that a decrease in the amount of homework completed by the client preceded an increase in the amount of cognitive changes, which in turn preceded a decrease in eating disorder symptomatology. It is important to note that assessments of cognitive change happened in session, whereas assessments of eating disorder symptomatology refer to the time in between sessions and client homework refer to their in session report of having completed homework during the week. 
points in therapy where clients will either continue to decrease homework completion and possibly fail in treatment or they will become more motivated, do more homework, and succeed in treatment.

\section{Similarity to Other Sudden Gains Findings}

The occurrence of sudden gains also appeared to follow the three-stage model proposed for sudden gain recovery in other disorders (i.e., cognitive changes during critical sessions $=>$ sudden gains $=>$ upward spiral that includes further cognitive changes and greater long-term symptom improvement) and specifically set in motion continued change or an "upward spiral" as noted by Tang and DeRubeis (1999b). In the treatment of eating disorders, the three stage model proposed for sudden gain recovery purports that after the pre-gain session, a positive feedback loop is triggered, as therapist cognitive interventions, relational interventions, client cognitive changes, and client motivation changes eventually lead to additional increases in levels of therapist empathy, client behavioral changes, client cognitive changes, and client motivation in the after-gain sessions. It can be speculated that this positive feedback loop might extend beyond the after-gain sessions and possibly lead these clients to sustained recovery.

When all of the client changes occurring between the pre-pregain, the pre-gain and postgain session are taken into account, a timeline of changes in some of the significant predictors can be created. The synchronized time courses of clients' cognitive changes, severity of eating disorder symptomatology, and behavioral changes (shown in Figure 7) indicate that increases in clients' cognitive change occurred first, followed immediately by the total CHEDS or Body subscale sudden gains. The sudden gains were, in turn, followed immediately by increases in client behavioral change and additional increases in cognitive change. It is likely that the behavioral focus in stage one and the identification of barriers for change in stage two allow 


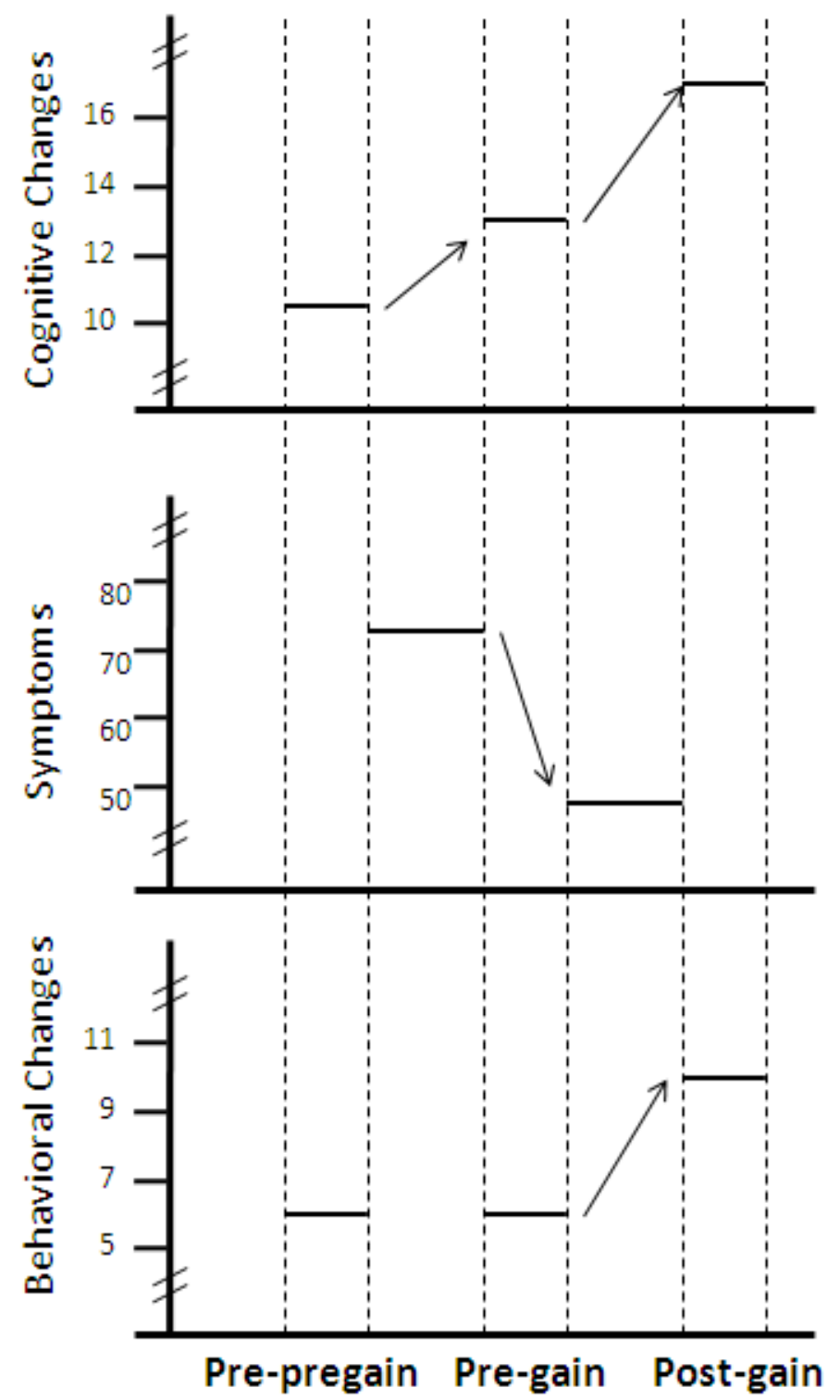

Figure 7. The synchronized time courses of client's cognitive changes, severity of eating disorder symptomatology, and behavioral changes demonstrates that an increase in the amount of cognitive changes preceded a decrease in eating disorder symptomatology, which in turn preceded an increase in both behavioral and cognitive changes. It is important to note that assessments of cognitive change and behavioral change were made from videotapes of each respective session, whereas assessments of eating disorder symptomatology refer to the time in between sessions. 
clients to experience some symptom reduction and to remove barriers that were preventing the possibility of a cognitive change happening. In summary, the increase in client cognitive change continues after the sudden gain happens, and client behavioral changes occur following the initial increase in cognitive change. There are many behavioral interventions used in this treatment that are effective in addressing behavioral symptoms that arise from the overvaluation of shape and weight, but given the finding that cognitive change predicted the appearance of both Total CHEDS and Body subscale sudden gains, it can be concluded that a core cognitive change in the client's over-evaluation of shape and weight is an important way of bringing about significant behavioral changes during the treatment of eating disorders.

In addition, similar to previous sudden gains findings, therapist empathy significantly increased following sudden gains and was not a predictor of sudden gains. This finding of relational variables improving after cognitive change and symptom improvement have taken place replicates previous findings (e.g. Tang \& DeRubeis, 1999b) that the therapeutic alliance can improve in response to rather than prior to positive therapeutic change. Additionally, in the present study, decreases in levels of therapist empathy were found to take place before and to predict eat subscale sudden gains. Thus, when an association between therapeutic alliance and outcome is observed, it does not automatically indicate that therapeutic alliance contributed to outcome.

\section{Strengths and Limitations}

\section{Strengths}

This study is the first to examine the existence of sudden gains in eating disorder treatment, contributing in a unique way to the study of symptom change patterns of eating disordered clients. The clients in this study were well defined as the diagnostic categories of 
bulimia nervosa and eating disorder not-otherwise specified. The treatment implemented was also well-specified and empirically supported for the treatment of eating disorders. Additionally, the therapists were well trained and supervised, there was a symptom change tracking measure administered at every session, and all sessions were videotaped. The measures used in this study are well established and psychometrically validated. In addition, sudden gains as defined in this study were identified using established indices of reliable change and clinical significance, which makes the definition of sudden gains equivalent to those used in sudden gains studies that examined disorders other than depression and it allows conclusions to be made about the statistical significance of clients' change scores and the change from a dysfunctional into a functional population.

\section{Limitations}

The diagnostic category of anorexia nervosa was unfortunately not included in this study. Even though there were a small number of anorexic clients included in Spangler's (2009) study, the established differences in those with anorexia nervosa in terms of treatment intervention and recovery pattern make inclusion of this group problematic. However, anorexia nervosa is part of the eating disorder spectrum and study findings will not be generalizable to this anorexic group. Self-selection of participants and attrition may affect the conclusions and generalizability of the findings of this study. Another possible limitation includes sample size. Although the initial sample size appears adequate, once sudden gains were identified and the initial group of clients was divided (thus decreasing the sample size by an expected 50\%), the likelihood of making an incorrect no-difference conclusion (Type II error) increases. This study only identified predictors of sudden gains that make the pre-gain session unique, not allowing causal relationships to be established between predictors and symptoms in their time course patterns 
across the duration of treatment. Finally, the three stage model of recovery proposed applies primarily to sudden-gain responders, that is, eating disorder clients who respond to CBT after experiencing sudden gains. It cannot address the mechanism of response for those who did not experience sudden gains. Indeed, it is possible that clients who responded to CBT without a sudden gain did so through different mechanisms or through similar mechanisms but with a more gradual course.

\section{Future Research}

The current study is comparable to previous work documenting the existence and clinical relevance of sudden gains and raises some other important questions surrounding the causes and effects of sudden gains in treatment. One aspect of the sudden gain phenomena that remains unexplored is the sudden gain-reversal. It is unclear what predicts, influences, or causes the sudden gain reversal. It would be useful to understand what takes place in the session right before the sudden gain reversal in the same manner that the current research investigated what took place in the session right before the sudden gain took place. The decrease in client homework that predicts the magnitude of total CHEDS and Body subscale sudden gains requires replication and further investigation, as it remains unknown why completion of homework drops before sudden gain. It may be due to struggles with treatment compliance, such as ambivalence about trusting the treatment, which could lead to these clients receiving higher levels of interventions aimed at addressing patient motivation for treatment, but future analyses of therapy sessions before the critical session are needed to test this hypothesis. In addition, given the notable outcome differences between clients who experienced sudden gains and those who did not, understanding what the difference is between those two groups seems important. It is possible that clients who responded to CBT without a sudden gain share some mechanisms of 
change with client who did respond to CBT with a sudden gain, but the more dissimilar mechanisms remain unclear.

\section{General Conclusion}

This study investigated whether sudden gains are present in eating disorders and whether they have significant implications for understanding the temporal recovery pattern from eating disorders. The existence of total CHEDS, Eat subscale, and Body subscale sudden gains and the determination that such gains are reliably stable and clinically significant fulfilled the first aim of the study and contributes to the understanding of the recovery trajectory of clients diagnosed with eating disorders who receive cognitive behavioral treatment. Both total CHEDS sudden gains and Body subscale sudden gains follow the three-stage model for sudden gain recovery wherein cognitive changes during a critical session lead to sudden notable symptom reduction , which in turn lead into an upward spiral that includes further cognitive changes, greater behavioral change, alliance improvement, and greater long-term symptom improvement. The present findings indicate that not only client cognitive change, but also therapist cognitive interventions, relational interventions, and client motivation initiate the three stage model. Therefore, there are both similarities, and differences between sudden gains in the treatment of eating disorders when compared to sudden gains in the treatment of other disorders. Predictors of sudden gains that distinguish the critical session from other therapy sessions were found for total CHEDS sudden gains, Body subscale sudden gains, and Eat subscale sudden gains indicating characteristics of critical therapy sessions and aspects of treatment that are important for clients' sudden gain recovery.

Combined with previous findings, the results of this study provide direct support for the cognitive mediation hypothesis of CBT. They revealed that in over 50\% of the treatment 
responders, superior treatment outcome was preceded by sudden gains, which were in turn immediately preceded by substantial cognitive interventions and cognitive changes in the pregain sessions. These significant differences among the pre-pregain, pre-gain, and post-gain sessions also demonstrate that CBT for eating disorders is not always a uniform and homogenous process. In particular, the pre-gain session is one type of critical session where changes in therapeutic events take place and help clients to progress in therapy. The nature of the therapy seems to have initially changed in the pre-gain session and to significantly continue to change after these sessions, strongly influencing the therapy outcome as a result. These findings regarding client in-session cognitive change not only further supports the cognitive mediation hypothesis, but also confirm that cognitive change is a key therapeutic factor in CBT for eating disorders. 


\section{References}

Agras, W. S., Crow, S. J., Halmi, K. A., Mitchell, J. E., Wilson, G. T., \& Kraemer, H. C. (2000). Outcome predictors for the cognitive behavior treatment of bulimia nervosa: Data from a multisite study. The American Journal of Psychiatry, 157(8), 1302-1308.

American Psychiatric Association (2000). Diagnostic and statistical manual of mental disorders ( $4^{\text {th }}$ ed). Washington, DC: American Psychiatric Association.

Andrusyna, T. P. (2008). The mechanism and time course of sudden gains in behavioral activation treatment for depression. ProQuest Information \& Learning). Dissertation Abstracts International: Section B: The Sciences and Engineering, 68 (9), 6287-6287.

Andrusyna, T. P., Luborsky, L., Pham, T., \& Tang, T. Z. (2006). The mechanisms of sudden gains in Supportive-Expressive therapy for depression. Psychotherapy Research, 16(5), 526536.

Barkham, M., Margison, F., Leach, C., Lucock, M., Mellor-Clark, J., Evans, C., et al. (2001). Service profiling and outcomes benchmarking using the CORE--OM: Toward practice-based evidence in the psychological therapies. Journal of Consulting \& Clinical Psychology, 69(2), 184.

Barlow, D. H. (2001). Clinical handbook of psychological disorders: A step-by-step treatment manual. New York: The Guilford Press.

Beck, A. T. (1979). Cognitive therapy and the emotional disorders. New York: International University Press.

Beck, A. T., Rush, A. J., Shaw, B. F., Emery, G. (1987). Cognitive therapy of depression. New York: The Guilford Press.

Beck, J. S. (1995). Cognitive therapy basics and beyond. New York: Guilford Press. 
Blackburn, I. M., Bishop, S., Glen, A. I., Whalley, L. J., \& Christie, J. E. (1981). The efficacy of cognitive therapy in depression: A treatment trial using cognitive therapy and pharmacotherapy, each alone and in combination. The British Journal of Psychiatry: The Journal of Mental Science, 139, 181-189.

Burns, D. D., \& Spangler, D. L. (2000). Does psychotherapy homework lead to improvements in depression in cognitive-behavioral therapy or does improvement lead to increased homework compliance? Journal of Consulting and Clinical Psychology, 68(1), 46-56.

Burns, D. D. (1989). The feeling good handbook. New York: Plume.

Busch, A. M., Kanter, J. W., Landes, S. J., \& Kohlenberg, R. J. (2006). Sudden gains and outcome: A broader temporal analysis of cognitive therapy for depression. Behavior Therapy, 37(1), 61-68.

Butler, A. C., Chapman, J. E., Forman, E. M., \& Beck, A. T. (2006). The empirical status of cognitive-behavioral therapy: A review of meta-analyses. Clinical Psychology Review, 26(1), $17-31$.

Clerkin, E. M., Teachman, B. A., \& Smith-Janik, S. B. (2008). Sudden gains in group cognitivebehavioral therapy for panic disorder. Behaviour Research and Therapy, 46(11), 1244-1250.

Cooper, Z., Cooper, P. J., \& Fairburn, C. G. (1989). The validity of the eating disorder examination and its subscales. The British Journal of Psychiatry: The Journal of Mental Science, $154,807-812$.

Cooper, Z., \& Fairburn, C. (1987). The eating disorder examination: A semi-structured interview for the assessment of the specific psychopathology of eating disorders. International Journal of Eating Disorders, 6(1), 1-8. 
Davies, L., Leach, C., Lucock, M., Stiles, W. B., Iveson, S., \& Barkham, M. (2006). Therapists' recall of early sudden gains in routine clinical practice. Psychology and Psychotherapy, 79, 107-114.

DeRubeis, R. J., Gelfand, L. A., Tang, T. Z., \& Simons, A. D. (1999). Medications versus cognitive behavior therapy for severely depressed outpatients: Mega-analysis of four randomized comparisons. The American Journal of Psychiatry, 156(7), 1007-1013.

Fairburn, C. G. (1981). A cognitive behavioural approach to the treatment of bulimia. Psychological Medicine, 11(4), 707-711.

Fairburn, C. G. (1995). Overcoming binge eating. New York: The Guilford Press.

Fairburn, C. G. (1997). Eating disorders. In D. M. Clark, C. G. Fairburn, D. M. Clark \& C. G. Fairburn (Eds.), Science and practice of cognitive behaviour therapy. (pp. 209-241). New York, NY US: Oxford University Press.

Fairburn, C. G. (2008). Cognitive behavioral therapy and eating disorders. New York: The Guilford Press.

Fairburn, C. G., Agras, W S., \& Wilson, G. T. (1992). The research on the treatment of bulimia nervosa: Practical and theoretical implications. In G. H. Anderson \& S. H. Kennedy (Eds.), The biology offeast and famine: Relevance to eating disorders (pp. 318-340). San Diego, CA: Academic Press.

Fairburn, C. G., Agras, W. S., Walsh, B. T., Wilson, G. T., \& Stice, E. (2004). Prediction of outcome in bulimia nervosa by early change in treatment. The American Journal of Psychiatry, 161(12), 2322-2324. 
Fairburn, C. G., \& Cooper, Z. (1993). The eating disorder examination (12th edition). In C. G. Fairburn, G. T. Wilson, C. G. Fairburn \& G. T. Wilson (Eds.), Binge eating: Nature, assessment, and treatment. (pp. 317-360). New York, NY US: Guilford Press.

Fairburn, C. G., Cooper, Z., Doll, H. A., O’Connor, M. A., Bohn, K., Hawker, D. M., Wales, J. A., \& Palmer, R. L. (2009). Transdiagnostic cognitive-behavioral therapy for patients with eating disorders: A two-site trial with 60-week follow-up. American Journal of Psychiatry, 166(3), 311-319.

Fairburn, C. G., Jones, R., Peveler, R. C., \& Carr, S. J. (1991). Three psychological treatments for bulimia nervosa: A comparative trial. Archives of General Psychiatry, 48(5), 463-469.

Fairburn, C. G., Marcus, M. D., \& Wilson, G. T. (1993). Cognitive-behavioral therapy for binge eating and bulimia nervosa: A comprehensive treatment manual. In C. G. Fairburn, G. T. Wilson, C. G. Fairburn \& G. T. Wilson (Eds.), Binge eating: Nature, assessment, and treatment. (pp. 361-404). New York, NY US: Guilford Press.

Feeley, M., \& DeRubeis, R. J. (1999). The temporal relation of adherence and alliance to symptom change in cognitive therapy for depression. Journal of Consulting \& Clinical Psychology, 67(4), 578.

Garner, D. M., Rockert, W., Davis, R., \& Garner, M. V. (1993). Comparison of cognitivebehavioral and supportive-expressive therapy for bulimia nervosa. American Journal of Psychiatry, 150(1), 37-46.

Gaynor, S. T., Weersing, V. R., Kolko, D. J., Birmaher, B., Heo, J., \& Brent, D. A. (2003). The prevalence and impact of large sudden improvements during adolescent therapy for depression: A comparison across cognitive-behavioral, family, and supportive therapy. Journal of Consulting and Clinical Psychology, 71(2), 386-393. 
Grencavage, L. M., \& Norcross, J. C. (1990). Where are the commonalities among the therapeutic common factors? Professional Psychology: Research and Practice, 21(5), 372378.

Hardy, G. E., Cahill, J., Stiles, W. B., Ispan, C., Macaskill, N., \& Barkham, M. (2005). Sudden gains in cognitive therapy for depression: A replication and extension. Journal of Consulting and Clinical Psychology, 73(1), 59-67.

Hofmann, S. G., Schulz, S. M., Meuret, A. E., Moscovitch, D. A., \& Suvak, M. (2006). Sudden gains during therapy of social phobia. Journal of Consulting \& Clinical Psychology, 74(4), 687-697.

Hollon, S. D., \& Kriss, M. R. (1984). Cognitive factors in clinical research and practice. Clinical Psychology Review, 4(1), 35-76.

Hwang, A. D., \& Spangler, D. L. (in progress). Classifying symptom change in eating disorders: Clinical significance metrics for the Change in Eating Disorder Symptom Scale.

Ilardi, S. S., \& Craighead, W. E. (1994). The role of nonspecific factors in cognitive-behavior therapy for depression. Clinical Psychology: Science and Practice, 1(2), 138-156.

Jacobson, N. S., \& Truax, P. (1991). Clinical significance: A statistical approach to defining meaningful change in psychotherapy research. Journal of Consulting and Clinical Psychology, 59(1), 12-19.

Jones, R., Peveler, R. C., \& Hope, R. A. (1993). Changes during treatment for bulimia nervosa: A comparison of three psychological treatments. Behaviour Research and Therapy, 31, 479485. 
Kelly, M. A. R., Roberts, J. E., \& Ciesla, J. A. (2005). Sudden gains in cognitive behavioral treatment for depression: When do they occur and do they matter? Behaviour Research and Therapy, 43(6), 703-714.

Kelly, M. A. R., Cyranowski, J. M., \& Frank, E. (2007). Sudden gains in interpersonal psychotherapy for depression. Behaviour Research and Therapy, 45(11), 2563-2572.

Leahy, R. L. (2001). Overcoming resistance in cognitive therapy. New York: Guilford CT.

Lewandowski, L. M., Gebing, T. A., Anthony, J. L., \& O'Brien, W. H. (1997). Meta-analysis of cognitive-behavioral treatment studies for bulimia. Clinical Psychology Review, 17(7), 703718.

Pham, T. D. (2006). The specificity and mechanism of sudden gains. ProQuest Information \& Learning). Dissertation Abstracts International: Section B: The Sciences and Engineering, $67(1), 556-556$.

Pham. T., Tang, T. Z., \& Andruzyna, T. (2004). Behavior change as triggers of sudden gains in moderate to severely depressed patients. Unpublished manuscript.

Pike, K. M., Devlin, M. J., \& Loeb, K. L. (2004). Cognitive-behavioral therapy in the treatment of anorexia nervosa, bulimia nervosa, and binge eating disorder. In J. K. Thompson, \& J. K. Thompson (Eds.), Handbook of eating disorders and obesity. (pp. 130-162). Hoboken, NJ US: John Wiley \& Sons Inc.

Present, J., Crits-Christoph, P., Gibbons, M. B. C., Hearon, B., Ring-Kurtz, S., Worley, M., et al. (2008). Sudden gains in the treatment of generalized anxiety disorder. Journal of Clinical Psychology, 64(1), 119-126.

Rosen, J. C. (1996). Body image assessment and treatment in controlled studies of eating disorders. International Journal of Eating Disorders, 20(4), 331-343. 
Shrout, P. E., \& Fleiss, J. L. (1979). Intraclass correlations: Uses in assessing rater reliability. Psychological Bulletin, 86, 420-428.

Spangler, D. L. (1999). Cognitive-behavioral therapy for bulimia nervosa: An illustration. Journal of Clinical Psychology, 55(6), 699-713.

Spangler, D.L. (in progress). The Change in Eating Disorder Symptom Scale: Scale development and psychometric properties. Manuscript in preparation.

Spangler, D. L., Baldwin, S. A., \& Agras, W. S. (2004). An examination of the mechanisms of action in cognitive behavioral therapy for bulimia nervosa. Behavior Therapy, 35(3), 537560.

Spangler, D. L., Beckstead, D. J., Hatch, A., Radpour-Wiley, M., \& Agras, W. S. (2001). Components of cognitive behavioral therapy: The factor structure of the cognitive coding scale for bulimia nervosa. Journal of Cognitive Psychotherapy, 15(4), 363-380.

Stiles, W. B., Barkham, M., Iveson, S., Iveson, M., Leach, C., Lucock, M., et al. (2003). Early sudden gains in psychotherapy under routine clinic conditions: Practice-based evidence. Journal of Consulting \& Clinical Psychology, 71(1), 14.

Tang, T. Z., \& DeRubeis, R. J. (1999a). Reconsidering rapid early response in cognitive behavioral therapy for depression. Clinical Psychology: Science and Practice, 6(3), 283-288.

Tang, T. Z., \& DeRubeis, R. J. (1999b). Sudden gains and critical sessions in cognitivebehavioral therapy for depression. Journal of Consulting \& Clinical Psychology, 67(6), 894.

Tang, T. Z., DeRubeis, R. J., Beberman, R., \& Pham, T. (2005). Cognitive changes, critical sessions, and sudden gains in cognitive--behavioral therapy for depression. Journal of Consulting \& Clinical Psychology, 73(1), 168-172. 
Tang, T. Z., deRubeis, R. J., Hollon, S. D., Amsterdam, J., \& Shelton, R. (2007). Sudden gains in cognitive therapy of depression and depression Relapse/Recurrence. Journal of Consulting \& Clinical Psychology, 75(3), 404-408.

Tang, T. Z., Luborsky, L., \& Andrusyna, T. (2002). Sudden gains in recovering from depression: Are they also found in psychotherapies other than cognitive-behavioral therapy? Journal of Consulting and Clinical Psychology, 70(2), 444-447.

Thackwray, D. E., \& Smith, M. C. (1993). A comparison of behavioral and cognitive-behavioral interventions for bulimia nervosa. Journal of Consulting \& Clinical Psychology, 61(4), 639.

Vittengl, J. R., Clark, L. A., \& Jarrett, R. B. (2005). Validity of sudden gains in acute phase treatment of depression. Journal of Consulting and Clinical Psychology, 73(1), 173-182.

Walsh, B. T., Wilson, G. T., Loeb, K. L., \& Devlin, M. J. (1997). Medication and psychotherapy in the treatment of bulimia nervosa. American Journal of Psychiatry, 154(4), 523-531.

Walter, S. D., Eliasziw, M., \& Donner, A. (1998). Sample size and optimal designs for reliability studies. Statistics in Medicine, 17,101.110.

Wampold, B. E. (2001). The great psychotherapy debate models, methods, and findings. Mahwah N.J.: L. Erlbaum Associates.

Whittal, M. L., \& Agras, W. S. (1999). Bulimia nervosa: A meta-analysis of psychosocial and pharmacological treatments. Behavior Therapy, 30(1), 117.

Wilson, G. T., \& Fairburn, C. G. (1993). Cognitive treatments for eating disorders. Journal of Consulting \& Clinical Psychology, 61(2), 261.

Wilson, G. T. (1999a). Rapid response to cognitive behavior therapy. Clinical Psychology: Science and Practice, 6(3), 289-292. 
Wilson, G. T. (1999b). Treatment of bulimia nervosa: The next decade. European Eating Disorders Review, 7(2), 77-83.

Wilson, G. T., Fairburn, C. C., Agras, W. S., Walsh, B. T., \& Kraemer, H. (2002). Cognitivebehavioral therapy for bulimia nervosa: Time course and mechanisms of change. Journal of Consulting and Clinical Psychology, 70(2), 267-274.

Wilson, G. T., \& Fairburn, C. G. (1993). Cognitive treatments for eating disorders. Journal of Consulting and Clinical Psychology, 61(2), 261-269.

Wilson, G. T., \& Fairburn, C. G. (1998). Treatments for eating disorders. In P. E. Nathan, J. M. Gorman, P. E. Nathan \& J. M. Gorman (Eds.), A guide to treatments that work. (pp. 501530). New York, NY US: Oxford University Press.

Wilson, G. T., \& Smith, D. (1989). Assessment of bulimia nervosa: An evaluation of the eating disorders examination. International Journal of Eating Disorders, 8(2), 173-179. 